\title{
UNIVERSALITY OF THE FUTURE CHRONOLOGICAL BOUNDARY
}

\author{
Steven G. HARRIS \\ CAPTIONS FOR ILLUSTRATIONS
}

Figure 1: IPs in $M$ and three embeddings for it Figure 2: spacetime with incomplete full causal boundary 


\title{
UNIVERSALITY OF THE FUTURE CHRONOLOGICAL BOUNDARY
}

\author{
Steven G. HARris
}

\begin{abstract}
The purpose of this note is to establish, in a categorical manner, the universality of the Geroch-Kronheimer-Penrose causal boundary when considering the types of causal structures that may profitably be put on any sort of boundary for a spacetime. Actually, this can only be done for the future causal boundary (or the past causal boundary) separately; furthermore, only the chronology relation, not the causality relation, is considered, and the GKP topology is eschewed. The final result is that there is a unique map, with the proper causal properties, from the future causal boundary of a spacetime onto any "reasonable" boundary which supports some sort of chronological structure and which purports to consist of a future completion of the spacetime. Furthermore, the future causal boundary construction is categorically unique in this regard.
\end{abstract}

\section{Section I: Introduction and Discussion}

The causal boundary of Geroch, Kronheimer, and Penrose [GKP] (also explicated in [HE], section 6.8) has played a role in helping to make clear the purely causal aspects of spacetimes, especially at timelike or null infinity. Being defined in terms of the pasts and futures of points, it seems to be a "natural" object for focussing on such matters. However, it has generally been considered somewhat opaque in application and difficult of calculation. Is it still worth pursuing in spite of its somewhat formidable reputation? An affirmative answer may be forthcoming if the intuitive sense of naturalness accruing to the causal boundary can be justified in a precise and general manner. It is the purpose of this note to do exactly that: to show that the causal boundary is a "universal object" in the strict categorical sense, that is to say, that in an appropriate mathematical category, the causal boundary construction is the essentially unique way of completing a spacetime in such a way as to yield a model to which any other causal completion process can naturally be compared (by means of a map from the causal boundary to the other boundary).

However, some caveats are in order: First of all, the categorical constructions performed here operate only on the future causal boundary (or, alternatively, only the past causal boundary), not on the melding together of the future and past boundaries that constitutes one of the more complicated issues in the full GerochKronheimer-Penrose (GKP) construction. Effectively, this is the portion of the GKP construction which places an endpoint on each future-endless causal curve in a spacetime, leaving past endpoints unaccounted for. Correspondingly, the categorical comparison will be made only with any "reasonable" future completion of a

This research was supported in part by the Department of Physics and Theoretical Physics of the Australian National University, and in part by NSF grant DMS9310477 to St. Louis University. 
spacetime - a completion of all future causal curves only. One way to understand why this is necessary is by noting that although the future causal boundary completes all future causal curves, and the past causal boundary completes all past causal curves, the combination of the two creates additional causal relations which can result in an object which is no longer complete in either future or past. (An example is given in the appendix.)

Second, in this presentation, only the chronology relation will be addressed, not the causality relation; this is more a matter of simplification than anything else: Categorical results can be obtained by working with only the one relation, so that is the path followed here, though there is no barrier to including both relations. Accordingly, the boundary construction here is called the future chronological boundary, allowing a distinction from the GKP (future) causal boundary.

Finally, all topology is eschewed from the approach here, with notions of convergence being treated by purely chronological means. This allows an enormous savings of complexity, as well as the avoidance of one of the more difficult elements of the GKP causal boundary. (A topology can be inferred from the chronological notion of convergence developed herein, but this will not play an explicit role.)

As a model for the construction followed here, consider the problem of completing a Riemannian manifold. There is more than one way to do this: One could take the one-point compactification, the Cauchy completion, or some arbitrary isometric embedding into a complete metric space. Part of the issue is that one is liable to end up with a non-manifold as one's complete object: The category of Riemannian manifolds and differentiable functions is not big enough to contain all the completions we may wish to consider. So the first thing to do is to expand to a larger category, the category Met of metric spaces and continuous functions. We'll use Cauchy completion as our method of completing not just any Riemannian manifold, but any metric space at all. This is actually a functor $\mathbf{C}:$ Met $\rightarrow$ Met $_{0}$ from Met to the subcategory of complete metric spaces, $\mathbf{M e t}_{0}$; this means that not only does each metric space $X$ have a Cauchy completion $\mathbf{C}(X)$, but also that for every map $f: X \rightarrow Y$ in Met, there is an extension of $f$ to the completions, $\mathbf{C}(f): \mathbf{C}(X) \rightarrow \mathbf{C}(Y)$, which works with compositions and with identity maps, i.e., $\mathbf{C}(f \circ g)=\mathbf{C}(f) \circ \mathbf{C}(g)$ and $\mathbf{C}\left(\operatorname{id}_{X}\right)=\operatorname{id}_{\mathbf{C}(X)}$. We need to take note not only of the completion of each metric space $X$ but also of its embedding into its completion, $i_{X}: X \rightarrow \mathbf{C}(X)$. This is a natural transformation $\mathbf{i}: \mathbf{I}_{\text {Met }} \dot{\rightarrow} \mathbf{U} \circ \mathbf{C}$, where $\mathbf{I}_{\text {Met }}$ is the identity functor on Met and $\mathbf{U}: \mathbf{M e t}_{0} \rightarrow$ Met is the "forgetful" (i.e., inclusion) functor; this means that for any map $f: X \rightarrow Y$, the embeddings on $X$ and $Y$ work with the extension to $f$, i.e., $i_{Y} \circ f=\mathbf{C}(f) \circ i_{X}$ (also, as a natural transformation, $i_{X}$ is considered as technically mapping $X$ to $\left.\mathbf{U}(\mathbf{C}(X))\right)$. The importance of the Cauchy construction for completion is that it is universal in the sense of being a left adjoint to the forgetful functor; this means that for any map from a metric space into a complete metric space, there is a unique extension of that map to the Cauchy completion: For any $X$ in Met, any $Y$ in $\mathbf{M e t}_{0}$, and any map $f: X \rightarrow \mathbf{U}(Y)$, there is a unique map $f_{0}: \mathbf{C}(X) \rightarrow Y$ such that $\mathbf{U}\left(f_{0}\right) \circ i_{X}=f$ (more simply, ignoring the forgetful functor: for any $f: X \rightarrow Y$ with $Y$ complete, there is a unique $f_{0}: \mathbf{C}(X) \rightarrow Y$ such that $\left.f_{0} \circ i_{X}=f\right)$. Since left adjoints are unique up to natural isomorphism (a natural transformation consisting of isomorphisms) ([M], Corollary IV.1), this tells us that Cauchy completion is the correct construction for the "free" completion of a metric space, in exact analogy with the 
free vector space on a set: It is functorial, natural, and universal. (But note that we had to expand from Riemannian manifolds to the larger setting of metric spaces in order to obtain a category in which the functoriality of the construction comes into play.)

The general idea here is to define a category of objects which includes both spacetimes and spacetimes that have boundaries attached to them, and to show that the future chronological boundary is a functorial, natural, and universal construction. The category, called chronological sets, consists, roughly speaking, of objects that are spacetime-like in that they possess a relation that works like the chronology relation in a spacetime. There is a great deal of freedom in choosing which properties of the spacetime relation to abstract and axiomatize for our new category. The ones chosen here seem to be the minimum ones needed to carry enough structure to be able to replace topology as the means for defining convergence for appropriate sequences of points. The approach here is somewhat reminiscent of the causal spaces axiomatized in $[\mathrm{KP}]$, and some of the language (such as "full" and "chain") from that paper is borrowed for this one; but there is a great deal of simplification achieved by concentrating solely on the chronology relation.

What is the practical impact of establishing the universality of the future chronological boundary? How does this help in understanding spacetimes? The answer lies in being able to relate any proposed future boundary for a spacetime to what is essentially the GKP future (pre-)boundary points, in a manner respecting the chronological relations - so long as the proposed boundary includes an extension of the chronology relation of the spacetime and does so in a "reasonable" manner (such as being past-distinguishing). Some examples at this point may help:

First an artificial example: Consider a spacetime $M$ obtained from Minkowski 2-space $\left(\mathbb{L}^{2}\right)$ by excising a timelike line segment $L$ ( such as $\{(0, t) \mid 0 \leq t \leq 1\}$ ). (A closely related example is considered in detail at the end of section IV.) The most obvious way to put a helpful boundary on $M$ is to embed it back into $\mathbb{L}^{2}$ in the obvious way, yielding $L$ as the boundary points for $M$. However, this is not the only useful possibility. Let us expand our conception of $M$ to include anything conformal to $\mathbb{L}^{2}-L$. Then, considering that the metric may become inherently singular as the points of $L$ are approached, we may wish to choose a boundary construction that respects $L$ as a physical blockage between its left and right sides; typically, we may choose as boundary two copies of $L$, one for the left side and one for the right side of the slit in $M$, joined at the top and the bottom, i.e., a boundary of the form $\left\{\left(0^{-}, t\right) \mid 0 \leq t \leq 1\right\} \cup\left\{\left(0^{+}, t\right) \mid 0 \leq t \leq 1\right\}$, with $\left(0^{-}, 0\right)$ and $\left(0^{+}, 0\right)$ identified, as well as $\left(0^{-}, 1\right)$ and $\left(0^{+}, 1\right)$. (By "physical blockage" here I refer to the chronology relation in the spacetime-cum-boundary: We have, for instance, $(-.1, .3) \ll\left(0^{-}, .5\right)$, and $(.1, .3) \ll\left(0^{+}, .5\right)$, but no chronology relation between $(-.1, .3)$ and $\left(0^{+}, .5\right)$. This reflects an impossibility of passage from one side of the slit to the other.) The GKP causal boundary produces precisely this latter boundary, in addition to the standard points at future and past null- and timelikeinfinity associated with Minkowski space. The future (pre-)boundary points of the GKP construction are similar, with the only difference being that there is a "double-point" at the top of the boundary: $\left(0^{-}, 1\right)$ and $\left(0^{+}, 1\right)$ are not identified but kept distinct. This last is the future chronological boundary.

What, then, does the universality of the future chronological boundary have to say in this example? It says that the future chronological boundary covers any 
other (past-distinguishing) boundary. For instance, the full GKP causal boundary identifies the double-point of the future chronological boundary into a single point. The first boundary mentioned, consisting of $L$ itself, identifies all the pairs of points $\left(0^{-}, t\right)$ and $\left(0^{+}, t\right)$. Other boundaries can be imagined, such as identifying $\left(0^{-}, t\right)$ and $\left(0^{+}, t\right)$ for some but not all $t$. (Note that past-distinguishment in the boundary necessitates retaining the identification of $\left(0^{-}, 0\right)$ and $\left(0^{+}, 0\right)$.) The point is that any appropriate boundary construction for $M$, intending to add future endpoints for all timelike curves, can be mapped onto by the future chronological boundary, in a manner that appropriately preserves the chronological relation; that this mapping is unique; and that the entire construction is categorical in nature.

For a more realistic example, let our spacetime be Schrwazschild space. The standard picture of the (future) singularity is of $\mathbb{S}^{2} \times \mathbb{R}^{1}$ : a 2-sphere (parametrized by $\phi$ and $\theta$ ) at each point of $r=0$ and any $t \in \mathbb{R}$, using Schwarzschild coordinates. We also have future null-infinity $\left(\Im^{+}=\mathbb{S}^{2} \times \mathbb{R}^{1}: r=\infty\right.$, other coordinates arbitrary) and future timelike-infinity $\left(i^{+}\right.$, a single point). What other reasonable options are there for future boundary points in Schwarzschild? The boundary just described is the future chronological boundary (external Schwarzschild is conformal to a standard static spacetime, which makes it easy to find its causal boundary). It follows that any past-distinguishing way of putting a future boundary on Schwarzschild must be a quotient of this: We can collapse the singularity to, for instance, a projective space or lens space at each value of $t$, and we can make identifications in $\Im^{+}$, but we cannot blow up $i^{+}$into a sphere or anything other than a single point.

It may be helpful to outline the some of the complexities encountered in this presentation.

The first step is to define a category which contains both reasonable (strongly causal) spacetimes and any such spacetime combined with a boundary of any sort (including an extension of the chronology relation to the boundary). The next step is to define a "future-completion" construction which will apply to any object in this category; it should replicate the GKP future (pre-)boundary construction when applied to spacetimes, and should yield nothing new when applied to an object which is already "future-complete". It is also intended that this future-completion construction not change anything in the original object, but just add additional points.

A problem arises in this, however: The condition used to define the the chronology relation for the new (future boundary) points - the same criterion as in the GKP construction - is not necessarily compatible with the pre-existing chronology relation in the original object. (Among spacetimes, global hyperbolicity prevents this from happening, but the idea is to provide a construction for all strongly causal spacetimes.) The problem is that if the same criterion is applied to the original object, then more pairs of points ought to be considered as chronologically related than was originally the case. For instance, if the object is the spacetime $M$ from the example above, then the criterion applied to $M$ would have $(-1,-1) \ll(.5,1)$; but there is no timelike curve between those points in $M$. Among other things, this incompatibility interferes with the functoriality of the future-completion construction.

The solution is to define an additional construction ("past-determination") on these objects whose sole purpose is to add in precisely those additional chronology relations so that when the future-completion construction is applied, the result is 
compatible with the chronology relation in the past-determined object. This defeats the intent of preserving the original chronology relation for the object, but there is no getting around this point. Indeed, this is precisely the tack chosen by GKP: The chronology relation in the GKP construction results in additional relations within the spacetime, exactly the same ones as the past-determination construction here. The exposition here essentially separates out this portion of the GKP construction for its own categorical and universal explication.

One still has to contend with the question of whether it makes a difference if one first past-determines and then future-completes, or does it the other way around. As it turns out, these operations are categorically commutative; but that takes some doing to establish.

Finally, one needs to make a comparison between the future chronological boundary construction here - the GKP future (pre-)boundary - and the full GKP causal boundary. A difficulty arises in that the simple definition of future-complete used here is not generally satisfied by the GKP causal boundary; a slight generalization is needed, allowing, for instance, for the identification of points such as in the cuasl boundary of the example $M$ above. But all goes through, as exemplified in that example.

In summation: Section II introduces the basic category and the future-completion operation; this applies, without further modification, to globally hyperbolic spacetimes. Section III defines the past-determination operation, extending the results of Section 1 to, for instance, all strongly causal spacetimes. Section IV addresses the generalization that is necessary in order to include such completions as those deriving from the full GKP construction.

A word on what this paper addresses and what it does not:

The intent here is to provide a justification for the use of the causal boundary construction. This is accomplished largely though showing how at least the future portion of that construction provides a universal standard of comparison for any other future-completing boundary, in tems of the chronology relation.

One obvious failing of this enterprise is to evince a similar standard of comparison for the full causal boundary. This, however, I believe to be innately impossible: As evidenced in the Appendix, the full causal boundary simply does not result in a complete object. This does not necessarily mean that the full causal boundary is an inappropriate tool, but it does detract from hopes of its having a universal role in boundary considerations.

Another element missing here is the causality relation. I believe it possible to do much of the same work here with the causality relation included, but it seems an unnecessary complication at this point. The chronology relation suffices to cover much of the necessary structures, and it yields a satisfactory universality result.

Topology is probably the most complex element missing from this development. It would be desireable to have a universality result which included full topological information, not just chronological. This is a matter under investigation and the subject for another paper.

\section{Section II: Chronological Sets and Future Completion}

The intent here is to abstract and axiomatize the chronology relation in a spacetime: A point $x$ chronologically precedes a point $y$, written $x \ll y$, if and only if 
there is a future-directed timelike curve from $x$ to $y$. This is the relation that must be emulated for the new category.

We start with a subset of the needed properties:

A pre-chronological set is a set $X$ together with a relation « satisfying the following conditions:

(1) $\ll$ is transitive: $x \ll y \ll z$ implies $x \ll z$

$(2) \ll$ is non-reflexive: for all $x, \operatorname{not}(x \ll x)$

(3) $X$ has no isolates: for all $x, I^{-}(x)=\{y \mid y \ll x\}$, the past of $x$, or $I^{+}(x)=$ $\{y \mid x \ll y\}$, the future of $x$, is non-empty

(4) « is full: for any $x \ll y$, there is some $z$ with $x \ll z \ll y$

For any non-empty subset $A$ of a pre-chronological set $X$, the past of $A, I^{-}[A]$, is $\bigcup_{a \in A} I^{-}(a)$ (the future of $A$, and all subsequent dualizations, are defined analogously). A non-empty subset $P$ is a past set if $I^{-}[P]=P$. (In general, ( ) will be used to indicate application of an operator to an entity to be regarded as a singleelement argument, [ ] will be used to indicate application to each of the various elements making up the argument.)

1. Proposition. In a pre-chronological set, any non-empty set of the form $I^{-}[A]$ is a past set.

Proof. By transitivity, $I^{-}\left[I^{-}[A]\right] \subset I^{-}[A]$. Now suppose $x \ll a$ for $a \in A$; then, by fullness, there is some $y$ with $x \ll y \ll a$. Thus, $I^{-}\left[I^{-}[A]\right]=I^{-}[A]$.

A past set is decomposable if it is the union of two proper subsets that are past sets. An indecomposable past set is often called an IP.

2. Theorem. In a pre-chronological set, a past set $P$ is indecomposable if and only if for every $x$ and $y$ in $P$, there is some $z \in P$ with $x \ll z$ and $y \ll z$.

Proof. (This theorem is due to Geroch, Kronheimer, and Penrose; the proof here is adapted from $[\mathrm{HE}]$, in the proof of Proposition 6.8.1.)

Suppose $P$ is decomposable as $P_{1} \cup P_{2}$ (proper past subsets). Then neither $P_{1} \subset P_{2}$ nor $P_{2} \subset P_{1}$, so we can find $x_{1} \in P_{1}-P_{2}$ and $x_{2} \in P_{2}-P_{1}$. Then there can be no $y \in P$ with $x_{1} \ll y$ and $x_{2} \ll y$ : If $y$ is in, say, $P_{1}$, then since $x_{2} \ll y$, $x_{2} \in P_{1}$ also, which is false.

Suppose $P$ is indecomposable. For any $x_{1}$ and $x_{2}$ in $P$, let $Q_{i}=\{q \in P \mid$ $\left.\operatorname{not}\left(x_{i} \ll q\right)\right\}$, and let $P_{i}=I^{-}\left[Q_{i}\right]$ (for each $i$ ). Note that $P_{i} \subset Q_{i}$ : For $x \in P_{i}$, there is some $q \in P$ with $x \ll q$ and not $\left(x_{i} \ll q\right)$; thus, $x \in P$ and $\operatorname{not}\left(x_{i} \ll x\right)$ (lest $x_{i} \ll q$ ), so $x \in Q_{i}$. Therefore, $P_{i}$ cannot be all of $P$, since $Q_{i}$ is not (since $x_{i}$ is in $P$ and $P=I^{-}[P]$, there must be some $p_{i} \in P$ with $x_{i} \ll p_{i}$ ). Since $P_{1}$ and $P_{2}$ are both past sets, it follows from $P$ being an IP that $P \neq P_{1} \cup P_{2}$, i.e., there is some $p \in P$ with $p \notin I^{-}\left[Q_{1}\right]$ and $p \notin I^{-}\left[Q_{2}\right]$. Now, since $P=I^{-}[P]$, there is some $z \in P$ with $p \ll z$; it then follows that $z$ must be in neither $Q_{i}$, i.e., that $x_{1} \ll z$ and $x_{2} \ll z$.

A future chain in a pre-chronological set $X$ is a sequence $\left\{x_{n} \mid n \geq 1\right\}$ of points in $X$ such that for all $n, x_{n} \ll x_{n+1}$.

A subset $S$ of a pre-chronological set $X$ is called dense in $X$ if for all $x$ and $y$ in $X$ with $x \ll y$, there is some $s$ in $S$ with $x \ll s \ll y . X$ is separable (in a chronological sense) if there is a countable dense set.

The following theorem shows how future chains in a chronological set replace future timelike curves in a spacetime; this is adapted from Proposition 6.8.1 in 
[HE], due to Geroch, Kronheimer, and Penrose, which makes essentially the same statement with "timelike curve" in the place of "chain".

3. Theorem. A past set in a separable pre-chronological set is indecomposable if and only if it is the past of a future chain.

Proof. Let $P$ be $I^{-}\left[\left\{x_{n} \mid n \geq 1\right\}\right]$ for $\left\{x_{n}\right\}$ a future chain; then for any $p_{1}$ and $p_{2}$ in $P$, there are numbers $n_{1}$ and $n_{2}$ with $p_{1} \ll x_{n_{1}}$ and $p_{2} \ll x_{n_{2}}$. Let $k=1+\max \left\{n_{1}, n_{2}\right\}$; then $x_{k}$ is in $P$ and has each $p_{i}$ in its past.

Let $P$ be an IP. If $S$ is the countable dense set for the pre-chronological set, then $P \cap S=\left\{p_{n} \mid n \geq 1\right\}$ is a dense set in $P$. Pick $x_{1} \ll p_{1}$ (one can always arrange to have the first element of $S$ have a non-empty past). Suppose we've defined $\left\{x_{1}, \ldots, x_{k}\right\}$ with $x_{i} \in P$ and $x_{i-1} \ll x_{i}$ and $p_{i} \ll x_{i}$ for each $i \leq k$. Then by Theorem 2 there is some $x_{k+1} \in P$ with $x_{k} \ll x_{k+1}$ and $p_{k+1} \ll x_{k+1}$. This defines'for us the future chain $\left\{x_{n}\right\}$ in $P$ with $p_{n} \ll x_{n}$ for each $n$. Clearly, $I^{-}\left[\left\{x_{n}\right\}\right] \subset P$. For any $x \in P$, since $P$ is a past set, there is some $y \in P$ with $x \ll y$; then there is some $k$ with $x \ll p_{k} \ll y$, so $p_{k} \ll x_{k}$ shows us $x \ll x_{k}$. Thus, $I^{-}\left[\left\{x_{n}\right\}\right]=P$.

We will say that an IP $P$ is generated by any future chain $c$ for which $P=I^{-}[c]$.

A pre-chronological set is called past-distinguishing if for points $x$ and $y, I^{-}(x)=$ $I^{-}(y)$ implies $x=y$. (Unlike nearly all the other terms defined here, this one is traditional, following the usage in $[\mathrm{HE}]$.)

For a future chain $c=\left\{x_{n} \mid n \geq 1\right\}$, a point $x$ is called a future limit of $c$ if $I^{-}(x)=I^{-}[c]$. Note that if $x$ is a future limit of $c$, then for all $n, x_{n} \ll x$, since $I^{-}(x)$ includes $I^{-}\left(x_{n+1}\right)$. Also, if $x$ is a future limit of $c$, then it is also a future limit of every sub-chain of $c$; and, conversely, if $x$ is a future limit of some sub-chain of $c$, then it is also a future limit of $c$. Clearly, in a past-distinguishing pre-chronological set, a future chain can have at most one future limit. (We could define a topology from this notion of convergence - a set is (future-) closed if it contains all future limits of future chains within it - but this will not be pursued here.)

A pre-chronological set that is separable is called a chronological set. A chronological set for which every future chain has a future limit is called future-complete.

It will prove useful to have the « relation determined, in a certain sense, by $I^{-}$: Specifically, call a pre-chronological set $X$ past-determined if for any $x$ and $y$ in $X$, if $I^{-}(x)$ is non-empty and for some $w \ll y, I^{-}(x) \subset I^{-}(w)$, then $x \ll y$. (We can also say that $x$ is past-determined if $I^{-}(x)$ is non-empty and whenever $I^{-}(x) \subset I^{-}(w), x \ll y$ for all $y \gg w$.)

4. Theorem. A strongly causal spacetime is a past-distinguishing chronological set; if it has the property that the past of $x$ is contained in the past of $y$ implies that $x$ causally precedes $y$ (for instance, any globally hyperbolic spacetime), then it is past-determined. A future limit of a future chain in a spacetime is the same as a topological limit.

Proof. A spacetime is clearly a pre-chronological set; it is separable in the chronological sense because it is separable in the topological sense. A strongly causal spacetime is past-distinguishing (see, e.g., [HE]).

If $I^{-}(x) \subset I^{-}(w)$ implies $x \in J^{-}(w)$ (points causally preceding $w$ ), then with $w \ll y$ also, we have $x \ll y$. Globally hyperbolic spacetimes are examples of this: For $I^{-}(x) \subset I^{-}(w)$, consider any $z \ll x$; then since $z \in I^{-}(x), z$ is also in $I^{-}(w)$, 
so $z \ll w$. It follows that the space $C(z, w)$ of causal curves from $z$ to $w$ is compact in the $C^{0}$ topology. Let $\sigma$ be a future timelike curve with $\sigma(0)=z$ and $\sigma(1)=x$; for all $t<1$, there is also a future timelike curve $\tau_{t}$ from $\sigma(t)$ to $w$. Let $c_{t}$ be the concatenation of $\left.\sigma\right|_{[0, t]}$ with $\tau_{t}$; then the family of causal curves $\left\{c_{t}\right\}$ has a causal limit curve $c_{1}$ in $C(z, w)$; since $c_{1}$ must pass through $x$, this shows $x \in J^{-}(w)$.

In a strongly causal spacetime, each point has a fundamental neighborhood system $\left\{U_{n}\right\}$ such that for each $n, U_{n}$ is geodesically convex and for any point $x \in U_{n}$, any other point $y \in U_{n}$ is timelike-related to $x$ iff it is joined to $x$ by a timelike geodesic in $U_{n}\left(x \ll y\right.$ but not joined by a timelike geodesic in $U_{n}$ implies that the timelike geodesic connecting them must exit and re-enter $U_{n}$, which can be prevented with correct choice of $\left.U_{n}\right)$. Let $c=\left\{x_{n}\right\}$ be a future chain. First suppose that $c$ has a topological limit $x$; then $c$ eventually enters all such neighborhoods $U_{n}$ of $x$ as just described. For any $y \ll x$, we have $x \in I^{+}(y)$, a neighborhood of $x$; thus, eventually, $x_{n} \in I^{+}(y)$, i.e., $y \ll x_{n}: I^{-}(x) \subset I^{-}[c]$. If any $x_{m}$ fails to be in the past of $x$, the same is true for all $x_{k}$ with $k>m$. Consider such an $x_{k}$ in a neighborhood $U_{n}$ of $x$ as above; due to the chronological relation in $U_{n}$ being given by the geodesic structure, this is essentially the same causal relations as exist in Minkowski space: In particular, $x_{k+1}$ is not in closure $\left(I^{-}(x)\right)$, and all $x_{j}$ for $j>k+1$ are in $I^{+}\left(x_{k+1}\right)$, whose closure does not include $x$. This violates $x$ being the limit of $c$; thus, we must have $I^{-}(x)=I^{-}[c]$. Conversely, suppose that $I^{-}(x)=I^{-}[c]$; then we have $x_{n} \ll x$ for each $n$ (since $x_{n} \in I^{-}[c]$ ). Using the neighborhoods $U_{n}$ of $x$ from above, we can find a past chain $\left\{y_{i}\right\}$ in $I^{+}(x)$ and a future chain $\left\{z_{i}\right\}$ in $I^{-}(x)$ with $x$ the topological limit of both chains and the sets $\left\{V_{i}=I^{-}\left(y_{i}\right) \cap I^{+}\left(z_{i}\right)\right\}$ a fundamental neighborhood system for $x$. Then for each $i$, since $z_{i} \in I^{-}(x)=I^{-}[c], x_{n} \gg z_{i}$ for sufficiently large $n$. Since $x_{n} \ll x \ll y_{i}$, this gives $x_{n} \in V_{i}$ for sufficiently large $n$, i.e., $x$ is the topological limit of $c$.

For a chronological set $X$, let $\hat{\partial}(X)=\{P \mid P$ is an IP in $X$ such that $P$ is not $I^{-}(x)$ for any point $\left.x \in X\right\}$, the future chronological boundary of $X$. Let $\hat{X}=$ $X \cup \hat{\partial}(X)$, the future chronological completion of $X$. Define an extension of $\ll$ on $X$ to $\hat{X}$ by the following:

(1) among points in $X$, there is no change in $\ll$

(2) for $x \in X$ and $P \in \hat{\partial}(X), x \ll P$ iff $x \in P$

(3) for $x \in X$ and $P \in \hat{\partial}(X), P \ll x$ iff for some $y \in X$ with $y \ll x, P \subset I^{-}(y)$

(4) for $P \in \hat{\partial}(X)$ and $Q \in \hat{\partial}(X), P \ll Q$ iff for some $y \in Q, P \subset I^{-}(y)$.

Where it is necessary to distinguish between the relation on $X$ and that on $\hat{X}, \hat{I}^{-}$ will be used for the latter. (Thus, $x \in \hat{I}^{-}(y)$ iff $x \in I^{-}(y)$, and so on.) Note that $\hat{I}^{-}(P)=P \cup\left\{Q \in \hat{\partial}(X) \mid Q \subset I^{-}(w)\right.$ for some $\left.w \in P\right\}=\hat{I}^{-}[P]$ (the first being the past of a single point in $\hat{X}$, the last being the past of a subset of $\hat{X}$ ); in particular, $\hat{I}^{-}(P) \cap X=P$. Also note that $P$ is a future limit of a future chain $c$ (in $\hat{X}$ ) if and only if $P$ is generated by $c\left(I^{-}[c]=P\right.$ iff $I^{-}[c]=I^{-}[P]$ iff $\hat{I}^{-}[c]=\hat{I}^{-}[P]$ iff $\left.\hat{I}^{-}[c]=\hat{I}^{-}(P)\right)$.

5. Theorem. Let $X$ be a chronological set. Then

(1) $\hat{X}$ is a chronological set;

(2) if $X$ is past-distinguishing, then so is $\hat{X}$;

(3) if $X$ is past-determined, then so is $\hat{X}$; and

(4) $\hat{X}$ is future-complete. 
Proof. (1) $\hat{X}$ is a chronological set:

This is a matter of routine checking of various cases. For instance, to show transitivity, one of the cases to be established is that for $x$ in $X$ and $P$ and $Q$ in $\hat{\partial}(X)$, if $x \ll P \ll Q$, then $x \ll Q$ : We have $x \in Q$ and $Q \subset I^{-}(w)$ for some $w \in R$; then $x \ll w$, and, since $R$ is a past set, $x \in R$, i.e. $x \ll R$.

A set which is dense in $X$ is dense in $\hat{X}$ as well (routine to establish).

Note that $\hat{X}$ has no isolates, since for any $P \in \hat{\partial}(X), \hat{I}^{-}(P)$ includes the elements of $P$, which is non-empty.

(2) If $X$ is past-distinguishing, then $\hat{X}$ is also $(x, P$, and $Q$ as above, with $y \in X)$ :

Suppose $\hat{I}^{-}(x)=\hat{I}^{-}(y)$. Then, in particular, $I^{-}(x)=I^{-}(y)$, so $x=y$.

Suppose $\hat{I}^{-}(x)=\hat{I}^{-}(P)$. Then, in particular, $\hat{I}^{-}(P) \cap X=I^{-}(x)$; but this means $P=I^{-}(x)$, which is forbidden by the definition of $\hat{\partial}(X)$.

Suppose $\hat{I}^{-}(P)=\hat{I}^{-}(Q)$. Then, in particular, $\hat{I}^{-}(P) \cap X=\hat{I}^{-}(Q) \cap X$, which just means precisely that $P=Q$.

(3) If $X$ is past-determined, then $\hat{X}$ is also (notation as above):

First note that if $\hat{I}^{-}(x)$ is non-empty, then so is $I^{-}(x)(P \ll x$ requires some $w \ll x)$, and $\hat{I}^{-}(P)$ is always non-empty.

Suppose $\hat{I}^{-}(x) \subset \hat{I}^{-}(w)$ with $w \ll y$; then $I^{-}(x) \subset I^{-}(w)$, so $x \ll y$. Suppose $\hat{I}^{-}(x) \subset \hat{I}^{-}(Q)$ with $Q \ll y$, i.e., $Q \subset I^{-}(w)$ and $w \ll y$; then $I^{-}(x) \subset Q$, so $I^{-}(x) \subset I^{-}(w)$, so $x \ll y$.

Suppose $\hat{I}^{-}(x) \subset \hat{I}^{-}(w)$ with $w \in R$ and $\hat{I}^{-}(x)$ non-empty; then we can find $z \in R$ with $w \ll z$, so the previous result gives us $x \ll z$, so $x \in R$, so $x \ll R$. Suppose $\hat{I}^{-}(x) \subset \hat{I}^{-}(Q)$ with $Q \subset I^{-}(w)$ and $w \in R$; then pick $z$ the same, and the previous result gives $x \ll z$, so $x \in R$, i.e., $x \ll R$.

Suppose $\hat{I}^{-}(P) \subset \hat{I}^{-}(w)$ with $w \ll y$; then $P \subset I^{-}(w)$, so $P \ll y$. Suppose $\hat{I}^{-}(P) \subset \hat{I}^{-}(Q)$ with $Q \subset I^{-}(w)$ and $w \ll y$; then $P \subset Q$, so $P \subset I^{-}(w)$, so $P \ll y$.

Suppose $\hat{I}^{-}(P) \subset \hat{I}^{-}(w)$ with $w \in R$; then pick $z \in R$ (i.e., $z \ll R$ ) with $w \ll z$, and the previous result yields $P \ll z$, so $P \ll R$. Suppose $\hat{I}^{-}(P) \subset \hat{I}^{-}(Q)$ with $Q \subset I^{-}(w)$ and $w \in R$ (i.e., $w \ll R$ ); then the previous result yields $P \ll w$, so $P \ll R$.

(4) $\hat{X}$ is future-complete:

First consider a future chain $c=\left\{x_{n}\right\}$ made up of points of $X$. Let $P=I^{-}[c]$; then $P$ is an IP in $X$. If there is a point $x \in X$ with $I^{-}(x)=P$, then $x$ is a future limit of $c$ : Since each $x_{n} \ll x$, for any $\alpha \in \hat{I}^{-}\left(x_{n}\right), \alpha \ll x$ also; for $y \in I^{-}(x)$, we have $y \in I^{-}[c]$; and for $Q \ll x$ with $Q \in \hat{\partial}(X)$, we have $Q \subset I^{-}(w)$ for some $w \ll x$, hence, $w \ll x_{n}$ for some $n$, so $Q \ll x_{n}$. Now suppose, on the other hand, that $P \in \hat{\partial}(X)$; then $P$ is a future limit of $c$, as noted previously.

Now consider a future chain $c$ in which there is some sub-chain $c^{\prime}$ consisting of points in $X$. By the paragraph above, $c^{\prime}$ has a future limit, which is then also a future limit for $c$.

For a future chain consisting solely of elements of $\hat{\partial} X$, just interpolate elements of $S$ (the countable dense subset of $X$ ) into the chain, resulting in a new future chain to which the previous paragraph applies; this has the same future limits as the original one. 
Let $f: X \rightarrow Y$ be a set map between pre-chronological sets. We will call $f$ chronological if $x \ll y$ in $X$ implies $f(x) \ll f(y)$ in $Y$; $f$ will be called futurecontinuous if, in addition, whenever $x$ is a future limit of a future chain $c$ in $X$, $f(x)$ is a future limit of $f[c]$ (necessarily a future chain in $Y$ ). For any chronological set $X$, we have the future-continuous map $\hat{\iota}_{X}: X \rightarrow \hat{X}$ given by $\hat{\iota}_{X}(x)=x$; this is the standard future injection of $X$ (that it is future-continuous follows directly from the definitions).

For $M$ and $N$ strongly causal spacetimes, a function $f: M \rightarrow N$ is both pastand future-continuous if and only if it is (topologically) continuous and carries timelike curves to timelike curves, preserving time-orientation. This can be seen by examining, for a point $x \in M$ with sequence $\left\{x_{n}\right\}$ topologically converging to $x$, a fundamental neighborhood system of the form described in the proof of Theorem $4,\left\{V_{i}=I^{-}\left(y_{i}\right) \cap I^{+}\left(z_{i}\right)\right\}$ for $\left\{y_{i}\right\}$ a past chain and $\left\{z_{i}\right\}$ a future chain, both with $x$ as topological limit. With $f$ both past- and future-continuous, we have $f(x)$ is the topological limit of the chains $\left\{f\left(y_{i}\right)\right\}$ and $\left\{f\left(z_{i}\right)\right\}$, so $\left\{W_{i}=\right.$ $\left.\left.I^{-}\left(f\left(y_{i}\right)\right) \cap I^{+} f\left(z_{i}\right)\right)\right\}$ is a fundamental neighborhood system for $f(x)$. Eventually, $x_{n} \in V_{i}$, whence $f\left(x_{n}\right) \in W_{i}$; thus, $f$ is topologically continuous. However, a merely future-continuous function need not be continuous: Consider $f: \mathbb{L}^{2} \rightarrow \mathbb{L}^{2}$ defined by $f(x, t)=(x, t)$ for $t>0$ and $f(x, t)=(x, t-1)$ for $t \leq 0\left(\mathbb{L}^{n}\right.$ denotes Minkowski $n$-space).

Let $f: X \rightarrow Y$ be a chronological map between chronological sets with $Y$ pastdistinguishing; we will define a map $\hat{f}: \hat{X} \rightarrow \hat{Y}$, the future completion of $f$. First we define $\bar{f}: \mathcal{P}(X) \rightarrow \mathcal{P}(Y)$ (the power sets) by $\bar{f}(S)=I^{-}[f[S]]$. For any point $x \in X$, we just let $\hat{f}(x)=f(x)$. For an IP $P \in \hat{\partial}(X)$, first note that $\bar{f}(P)$ is an IP in $Y$ : This is because for any future chain $c$ generating $P, \bar{f}(P)=I^{-}[f[c]]$. If there is some $y \in Y$ with $I^{-}(y)=\bar{f}(P)$, then we let $\hat{f}(P)=y$; otherwise, we let $\hat{f}(P)=\bar{f}(P)$. (Note that $\hat{f}$ can be defined even if $Y$ is not past-distinguishing, so long as there is never more than a single point $y \in Y$ for which $I^{-}(y)$ is the same as a given $\bar{f}(P)$.)

6. Proposition. Let $f: X \rightarrow Y$ be a chronological map between chronological sets with $Y$ past-determined and past-distinguishing (or, more generally: for any future chain in $X$ with no future limit in $X$, its image under $f$ does not have more than one future limit in $\hat{Y}$ ); then $\hat{f}$ is also chronological. If $f$ is future-continuous, then $\hat{f}$ is the unique future-continuous map such that $\hat{f} \circ \hat{\iota}_{X}=\hat{\iota}_{Y} \circ f$.

Proof. That $\hat{f} \circ \hat{\iota}_{X}=\hat{\iota}_{Y} \circ f$ is clear. From this and $f$ being chronological it follows that $x \ll y$ in $X$ implies $\hat{f}(x) \ll \hat{f}(y)$. Suppose $x \ll P$ for $x \in X$ and $P \in \hat{\partial}(X)$. Let $P$ be generated by a future chain $c=\left\{x_{n}\right\}$, so that $f[c]$ generates $\bar{f}(P)$. We have $x \in P$, so $x \ll x_{n}$ for some $n$; thus, $f(x) \ll f\left(x_{n}\right)$, so $f(x) \in \bar{f}(P)$. If $\bar{f}(P) \in \hat{\partial}(Y)$, this is all we need for $\hat{f}(x) \ll \hat{f}(P)$; otherwise, for some $y \in Y, \bar{f}(P)=I^{-}(y)$, so $f(x) \in I^{-}(y), \operatorname{orM} f(x) \ll y$, so $\hat{f}(x) \ll \hat{f}(P)$. If $P \ll x$ (same notation), then $P \subset I^{-}(w)$ for some $w \ll x$. With the future chain $c$ as before, we have for all $n, x_{n} \ll w \ll x$, so $f\left(x_{n}\right) \ll f(w) \ll f(x)$. This gives us $\bar{f}(P) \subset I^{-}(f(w))$. If $\bar{f}(P) \in \hat{\partial}(Y)$, then $\hat{f}(P) \ll \hat{f}(x)$; otherwise, for some $y \in Y, \bar{f}(P)=I^{-}(y)$, so $y \ll f(x)$ by $Y$ being past-determined. Finally, suppose $P \ll Q$ for $P$ and $Q$ in $\hat{\partial}(X)$; then there is some $x \in X$ with $P \ll x \ll Q$, so the previous results apply.

Suppose $f$ is future-continuous. Clearly, for a point $x \in X$ being a future limit of a future chain of points $c=\left\{x_{n}\right\}$ in $X$, the same is true for $\hat{f}(x)$ and $\left\{\hat{f}\left(x_{n}\right)\right\}$. 
For $P \in \hat{\partial}(X)$ being a future limit of the chain, since $P$ is generated by $c, \bar{f}(P)$ is generated by $f[c]$. Thus, if $\bar{f}(P) \in \hat{\partial}(Y)$, then it is the future limit of $f[c]$, and we are done, since $\hat{f}(P)=\bar{f}(P)$. Otherwise, for some $y \in Y, \bar{f}(P)=I^{-}(y)$ and again we are done, since $\hat{f}(P)=y$ and $y$ is a future limit of $f[c]=\hat{f}[c]$. We need concern ourselves solely with chains of points in $X$, for any other sort can have points of $X$ interpolated into it with no change in future limits.

The restriction of $\hat{f}$ to $X$ is uniquely determined by $\hat{f} \circ \hat{\iota}_{X}=\hat{\iota}_{Y} \circ f$. For $\hat{f}$ to be future-continuous, for any $P \in \hat{\partial}(X)$, since $P$ is a future limit of any chain $c$ generating it, we must have $\hat{f}(P)$ a future limit of $f[c]$. With $Y$ past-distinguishing (or, more generally, with the definition of $\hat{f}$ above yielding a well-defined function), this is unique.

Another way to express the previous result is more closely related to adjunctions:

7. Corollary. Let $f: X \rightarrow Y$ be a future-continuous function between chronological sets with $Y$ past-determined, past-distinguishing, and future-complete (or, more generally: for any future chain in $X$, its image under $f$ has exactly one future limit in $Y$, which is past-determined); then there is a unique future-continuous function $\hat{f}: \hat{X} \rightarrow Y$ such that $\hat{f} \circ \hat{\iota}_{X}=f$.

Proof. We need but note that with $Y$ being future complete, $\hat{Y}=Y$ and $\hat{\iota}_{Y}$ is the identity map on $Y$; then Proposition 6 applies.

Conversely, we could have started with Corollary 7 and deduced Proposition 6: Given future-continuous $f: X \rightarrow Y$ with $Y$ past-determined and past-distinguishing, we have $\hat{\iota}_{Y} \circ f: X \rightarrow \hat{Y}$ fulfilling the hypotheses of Corollary 7, yielding $\hat{f}: \hat{X} \rightarrow \hat{Y}$ as the unique future-continuous map such that $\hat{f} \circ \hat{\iota}_{X}=\hat{\iota}_{Y} \circ f$.

These results allow us to define some categories, some functors, a natural transformation, and an adjunction: Let Chron be the category of chronological sets with morphisms all chronological functions; since the composition of chronological functions is clearly chronological, this is a category. Let PdetPdisChron be the subcategory of past-determined, past-distinguishing chronological sets, with morphisms all future-continuous functions; again, a composition of future-continuous functions is future-continuous, so this is a category. Let FcplPdetPdisChron be the full subcategory of PdetPdisChron of objects which are also future-complete. Then future completion is a functor ${ }^{\wedge}:$ PdetPdisChron $\rightarrow$ FcplPdetPdisChron (we need only check that $\widehat{g \circ f}=\hat{g} \circ \hat{f}$; this follows from the uniqueness part of Proposition 6 , since $\hat{g} \circ \hat{f}$ has the requisite properties); while Theorem 5 gives us the right properties for the future completion of any chronological set, Proposition 6 gives us functoriality only for this subcategory. We also have the forgetful functor $\hat{\mathbf{U}}: \mathbf{F c p l P d e t P d i s C h r o n} \rightarrow$ PdetPdisChron. The maps $\hat{\iota}_{X}$ define a natural transformation $\hat{\boldsymbol{\iota}}: \mathbf{I} \rightarrow \hat{\mathbf{U}} \circ^{-}$(where $\mathbf{I}$ is the identity functor on PdetPdisChron). Finally, Proposition 7 gives us the universality condition on $\hat{\boldsymbol{\iota}}$ making $\widehat{\wedge}$ left adjoint to $\hat{\mathbf{U}}$. From category theory we obtain

8. Theorem. The future completion functor and the standard future injection are, up to natural isomorphism, the unique construction yielding a future-continuous map from any past-determined, past-distinguishing chronological set to a futurecomplete, past-determined, past-distinguishing chronological set, consistent with all future-continuous maps and yielding a unique extension for any future-continuous 
map into a future-complete, past-determined, past-distinguishing chronological set.

To restate the previous three theorems in summary form:

(1) For $X$ in Chron, $Y$ in PdetPdisChron, and $f: X \rightarrow Y$ future-continuous, $\hat{f}: \hat{X} \rightarrow \hat{Y}$ is a future-continuous map with $\hat{f} \circ \hat{\iota}_{X}=\hat{\iota}_{Y} \circ f$.

(2) For $X$ in Chron, $Y$ in FcplPdetPdisChron, and $f: X \rightarrow Y$ futurecontinuous, $\hat{f}: \hat{X} \rightarrow Y$ is the unique future-continuous map with $\hat{f} \circ \hat{\iota}_{X}=f$.

(3) Restricted to PdetPdisChron, the construction of $\hat{X}, \hat{f}$, and $\hat{\iota}_{X}$ is functorial, natural, and universal, hence, unique as a way to fulfill (1) and (2) in such a manner.

\section{Section III: Past-Determination Functor}

It is quite common for a spacetime not to be past-determined, so there is a limited application of the functor ${ }^{\wedge}$ to spacetimes. However, this can be remedied if we are willing to create new « relations in the spacetime, in order to make it past-determined. We will do this generally:

Consider a pre-chronological set $X$; using the same set $X$, let us define a new relation, $\ll^{p}$ : For any $x$ and $y$ in $X$, set $x \ll^{p} y$ if $x \ll y$ or if $I^{-}(x)$ is non-empty and for some $w \ll y, I^{-}(x) \subset I^{-}(w)$; we'll use $I^{-p}$ to denote the past of a point (or set) using the $\ll^{p}$ relation. Clearly, $\ll^{p}$ is an extension of $\ll$. Note that if $X$ is past-determined, then $\ll^{p}$ is identical to $\ll$.

Two properties of $\ll^{p}$ should be noted: First, for any $x, I^{-}\left[I^{-p}(x)\right]=I^{-}(x)$ : If $y \ll z \ll^{p} x$, then $z \ll x$ (and so $y \ll x$ ) or $I^{-}(z) \subset I^{-}(w)$ for some $w \ll x$; we have $y \in I^{-}(z)$, so we get $y \in I^{-}(w)$, i.e., $y \ll w$, so $y \ll x$. On the other hand, if $y \ll x$, then by fullness there is some $z$ with $y \ll z \ll x$; this gives us $z \ll^{p} x$, so $y \in I^{-}\left[I^{-p}(x)\right]$. Second, $I^{-}(x) \subset I^{-}(y)$ if and only if $I^{-p}(x) \subset I^{-p}(y)$ : Given $I^{-}(x) \subset I^{-}(y)$, if $z \ll^{p} x$, then we have $z \ll x$ (so $\left.z \in I^{-}(x) \subset I^{-}(y) \subset I^{-p}(y)\right)$ or we have $I^{-}(z) \neq \emptyset$ and $I^{-}(z) \subset I^{-}(w)$ for some $w \ll x$; in the latter case, we have $w \ll y$ also, so $z \ll^{p} y$. On the other hand, given $I^{-p}(x) \subset I^{-p}(y)$, we obtain, from the first property noted, $I^{-}(x)=I^{-}\left[I^{-p}(x)\right] \subset I^{-}\left[I^{-p}(y)\right]=I^{-}(y)$. In particular, we have $I^{-}(x)=I^{-}(y)$ if and only if $I^{-p}(x)=I^{-p}(y)$.

For a spacetime, the physical significance of the $\ll^{p}$ relation can be viewed this way: Consider any observer as compiling an encyclopedia of all events witnessed; the spacetime is to be thought of also as the set of encyclopedias, with the event $p$ corresponding to the history of all events in $I^{-}(p)$. Then $p \ll^{p} q$ means that not only has $q$ transcribed all the events that are written at $p$, but, moreover, has had a chance to think about them (i.e., an observer has traveled from some event $w$, which has recorded all events that are written at $p$, and then continued on to event $q)$.

We will use $X^{p}$ to denote $X$ with the relation $\ll^{p}$; call it the past-determination of $X$.

If $c=\left\{x_{n}\right\}$ is a future chain in $X^{p}$, then we have for all $n, I^{-}\left(x_{n}\right) \subset I^{-}\left(w_{n}\right)$ for some sequence $\left\{w_{n}\right\}$ with $w_{n} \ll x_{n+1}$. This gives us $w_{n} \in I^{-}\left(x_{n+1}\right) \subset I^{-}\left(w_{n+1}\right)$, so $w_{n} \ll w_{n+1}$ : $c^{\prime}=\left\{w_{n}\right\}$ is a future chain in $X$. Note that $I^{-}\left[c^{\prime}\right]=I^{-}[c]$, due to the interweavings $I^{-}\left(x_{n}\right) \subset I^{-}\left(w_{n}\right)$ and $I^{-}\left(w_{n}\right) \subset I^{-}\left(x_{n+1}\right)$; in general, when this interweaving obtains, we will say that $c^{\prime}$ (in $X$ ) is an associated chain to $c$ (in $\left.X^{p}\right)$. This works well for looking at future limits: 
9. Lemma. Let $c^{\prime}$ be a future chain in $X$ associated to a future chain $c$ in $X^{p}$. Then a point $x$ is a future limit of $c$ in $X^{p}$ if and only if it is a future limit of $c^{\prime}$ in $X$.

Proof. Suppose $I^{-p}(x)=I^{-p}[c]$; then $I^{-}(x)=I^{-}[c]=I^{-}\left[c^{\prime}\right]$. Now suppose $I^{-}(x)=I^{-}\left[c^{\prime}\right]$; then $I^{-}(x)=I^{-}[c]$. Let $c=\left\{x_{n}\right\}$. For $z \ll^{p} x$, we have $I^{-}(z)$ is non-empty and $I^{-}(z) \subset I^{-}(w)$ for some $w \ll x$; since $I^{-}(x)=I^{-}[c]$, we have $w \ll x_{n}$ for some $n$, so $z \in I^{-p}[c]$. On the other hand, if $z \in I^{-p}[c]$, then $I^{-}(z)$ is non-empty and $I^{-}(z) \subset I^{-}(w)$ for some $w \ll x_{n}$ for some $n$; then (since $I^{-}[c]=$ $\left.I^{-}(x)\right) w \ll x$ also, so $z \ll^{p} x$.

10. Proposition. For any pre-chronological set $X, X^{p}$ is a past-determined prechronological set. If $X$ is a chronological set, so is $X^{p}$; if $X$ is past-distinguishing, so is $X^{p}$; and if $X$ is future-complete, so is $X^{p}$.

Proof. This is largely routine. For example, to show $X^{p}$ is past-determined, consider $I^{-p}(x) \subset I^{-p}(w)$ with $I^{-p}(x)$ non-empty and $w \ll^{p} y$; we want to show $x \ll^{p} y$. From $w \ll^{p} y$ we know that for some $v \ll y, I^{-}(w) \subset I^{-}(v)$ (this is true even if $w \ll y)$. We have $I^{-}(x) \subset I^{-}(w)$, so $I^{-}(x) \subset I^{-}(v)$. Since $I^{-p}(x)$ is non-empty, so is $I^{-}(x)$. Thus, $x \ll^{p} y$.

Suppose $X$ is future-complete. Let $c=\left\{x_{n}\right\}$ be a future chain in $X^{p}$. Let $x$ be a future limit of an associated future chain $c^{\prime}$ in $X$; then by Lemma $9, x$ is also a future limit of $c$ in $X^{p}$.

Past-determination works well with future-continuous functions, so long as the domain has the property that every point is a future limit of some future chain; let us call this property being past-connected. Clearly, any spacetime is past-connected, and future completion preserves being past-connected (since an element of the future chronological boundary is always a future limit of any of its generating chains). Also, forming the past-determination of a pre-chronological set preserves being pastconnected, in virtue of Lemma 9.

11. Proposition. If $f: X \rightarrow Y$ is a future-continuous map between pre-chronological sets, and $X$ is past-connected, then the same function $f^{p}=f: X^{p} \rightarrow Y^{p}$ between the past-determinations is also future-continuous.

Proof. First we will show that $f^{p}$ is chronological; we must show that if $x \ll^{p} y$, then $f(x) \ll^{p} f(y)$ : We have $I^{-}(x)$ is non-empty and $I^{-}(x) \subset I^{-}(w)$ for some $w \ll y$. We have some $z \ll x$, so $f(z) \ll f(x)$ : $I^{-}(f(x))$ is non-empty. With $x$ a future limit of a future chain $c, f(x)$ is a future limit of $f[c]$, so $I^{-}(f(x))$ $=I^{-}[f[c]]$. We have $c \subset I^{-}(w)$, so $f[c] \subset I^{-}(f(w))$, so $I^{-}[f[c]] \subset I^{-}(f(w))$. Therefore, $I^{-}(f(x)) \subset I^{-}(f(w))$ and $f(w) \ll f(y): f(x) \ll^{p} f(y)$.

Now suppose that $c=\left\{x_{n}\right\}$ is a future chain in $X^{p}$ with future limit $x$. For $c^{\prime}$ an associated chain in $X$, we know by Lemma 9 that $x$ is also a future limit in $X$ of $c^{\prime}$; then $f(x)$ is a future limit in $Y$ of $f\left[c^{\prime}\right]$. Furthermore, $f\left[c^{\prime}\right]$ is an associated chain of $f[c]$ (the relations'defining association are preserved by a chronological function), so Lemma 9 also gives us that $f(x)$ is a future limit in $Y^{p}$ of $f[c]$.

For any pre-chronological set $X$, let us denote by $\iota_{X}^{p}: X \rightarrow X^{p}$ the map which, on the set level, is the identity map. This is clearly chronological, and it is also future-continuous: If, for a future chain $c$ and point $x$ in $X, I^{-}(x)=I^{-}[c]$, then $I^{-p}(x)=I^{-p}[c]$ also (using the second property of $\ll^{p}$ mentioned at the beginning of this section). 
12. Corollary. Let $f: X \rightarrow Y$ be a future-continuous map between pre-chronological sets, with $X$ past-connected and $Y$ past-determined; then there is a unique future-continuous map $f^{p}: X^{p} \rightarrow Y$ such that $f^{p} \circ \iota_{X}^{p}=f$.

Proof. By Proposition 10, $Y^{p}=Y$. Then the map $f^{p}: X^{p} \rightarrow Y^{p}$ given by Proposition 11 fills the required condition, and uniquely does so because of what $\iota_{X}^{p}$ is on the set level.

Proposition 11 and Corollary 12 have the same categorical properties as Proposition 6 and Corollary 7, and we derive similar categorical constructions: We have a functor $\mathbf{p}$ : PconChron $\rightarrow$ PdetPconChron, where -Pcon- denotes the subcategory of past-connected objects. We also have a natural transformation $\iota^{\mathbf{p}}: \mathbf{I} \rightarrow \mathbf{U}^{\mathbf{p}} \circ \mathbf{p}$, where $\mathbf{U}^{\mathbf{p}}: \mathbf{P d e t P c o n C h r o n} \rightarrow$ PconChron is the forgetful functor and $\mathbf{I}$ is the identity functor on PconChron. Then Corollary 12 is precisely the statement that $\mathbf{p}$ (via $\iota^{\mathbf{p}}$ ) is a left adjoint to $\mathbf{U}^{\mathbf{p}}$. (The same applies to the restriction, in all cases, to the respective subcategories of past-distinguishing objects, -Pdis-, which will be used for composition with the future completion functor.) Thus, similar to Theorem 8 , we can conclude that the past-determination functor is the unique way to create a past-determined chronological set (at least, if one starts with all points being future limits), in a categorical, natural, and universal manner. Summarizing as before, we have

(4) For $X$ in PconPrechron, $Y$ in Prechron, and $f: X \rightarrow Y$ futurecontinuous, $f^{p}: X^{p} \rightarrow Y^{p}$ is the unique future-continuous map with $f^{p} \circ \iota_{X}^{p}=\iota_{Y}^{p} \circ f$.

(5) For $X$ in PconPrechron, $Y$ in PdetPrechron, and $f: X \rightarrow Y$ futurecontinuous, $f^{p}: X^{p} \rightarrow Y$ is the unique future-continuous map with $f^{p} \circ \iota_{X}^{p}=$ $f$.

(6) Restricted to PconChron (or, more generally, PconPrechron), the construction of $X^{p}, f^{p}$, and $\iota_{X}^{p}$ is functorial, natural, and universal, hence, the unique way to fulfill (4) and (5) in such a manner. The same is true for the further restriction to PconPdisChron.

We can combine these results with those of the previous section, composing the categorical items: Restricting the functor ${ }^{-}$to PdetPconPdisChron, we have the composition ${ }^{\wedge} \circ \mathbf{p}:$ PconPdisChron $\rightarrow$ FcplPdetPconPdisChron and the forgetful functor $\mathbf{U}^{+}=\hat{\mathbf{U}} \circ \mathbf{U}^{\mathbf{P}}$ in the opposite direction. For any $X$ in PconChron, let $\iota_{X}^{\uparrow}=\hat{\iota}_{X^{p}} \circ \iota_{X}^{p}: X \rightarrow \widehat{X^{p}}$; then the collection of maps $\left\{\iota_{X}^{\uparrow}\right\}$ forms a natural transformation $\boldsymbol{\iota}^{\uparrow}: \mathbf{I} \rightarrow \mathbf{U}^{+} \circ^{\wedge} \circ \mathbf{p}(\mathbf{I}$ as above $)$, and this yields ${ }^{\wedge} \circ \mathbf{p}$ as left adjoint to $\mathbf{U}^{+}$. Summarizing as before, we have

(7) For $X$ in PconChron, $Y$ in PdisChron, and $f: X \rightarrow Y$ futurecontinuous, $\widehat{f^{p}}: \widehat{X^{p}} \rightarrow \widehat{Y^{p}}$ is the unique future-continuous map with $\widehat{f^{p}} \circ \iota_{X}^{\uparrow}=\iota_{Y}^{\uparrow} \circ f$.

(8) For $X$ in PconChron, $Y$ in FcplPdetPdisChron, and $f: X \rightarrow Y$ futurecontinuous, $\widehat{f^{p}}: \widehat{X^{p}} \rightarrow Y$ is the unique future-continuous map with $\widehat{f^{p}} \circ \iota_{X}^{\uparrow}=$ $f$.

(9) Restricted to PconPdisChron, the construction of $\widehat{X^{p}}, \widehat{f^{p}}$, and $\iota_{X}^{\uparrow}$ is functorial, natural, and universal, hence, the unique way to fulfill (7) and (8) in such a manner.

We need to consider how past-determination interacts with future completion. 
First, let us compare the past of a point $x$ of $X$ in $\hat{X}^{p}$ with its past in $\widehat{X}^{p}$ : For $y \in X$, we have $y \in \hat{I}^{-p}(x)$ if $y \ll x$ or if $\hat{I}^{-}(y)$ is non-empty and $\hat{I}^{-}(y) \subset \hat{I}^{-}(w)$ for some $w \ll x$; while we have $y \in \widehat{I^{-p}}(x)$ if $y \ll x$ or if $I^{-}(y)$ is non-empty and $I^{-}(y) \subset I^{-}(w)$ for some $w \ll x$. These are equivalent conditions: For instance, suppose $I^{-}(y) \subset I^{-}(w)$; then $P \ll y$ means $P \subset I^{-}(z)$ for some $z \ll y$, which then yields $z \ll w$, so $P \ll w$, also. For $Q \in \hat{\partial}(X)$, we have $Q \in \hat{I}^{-p}(x)$ if $Q \ll x-$ i.e., if $Q \subset I^{-}(w)$ for some $w \ll x$-or if $\hat{I}^{-}(Q)$ is non-empty and $\hat{I}^{-}(Q) \subset \hat{I}^{-}(w)$ for some $w \ll x$; while for $Q \in \hat{\partial}\left(X^{p}\right)$, we have $Q \in \widehat{I^{-p}}(x)$ if $Q \subset I^{-p}(w)$ for some $w \ll^{p} x$. But to compare these two conditions directly is awkward, and we'll instead use a pair of maps to aid in the analysis.

For a chronological set $X$, let us define the maps $i: \hat{X}^{p} \rightarrow \widehat{X^{p}}$ and $j: \widehat{X^{p}} \rightarrow \hat{X}^{p}$ as follows: Both maps are to be the identity on $X$. For $P \in \hat{\partial}(X)$, let $c$ be any future chain in $X$ generating $P$; then $i(P)=I^{-p}[c]$. For $Q \in \hat{\partial}\left(X^{p}\right)$, let $c$ be a future chain in $X^{p}$ generating $Q$; let $c^{\prime}$ be an associated chain in $X$, and let $j(Q)=I^{-}\left[c^{\prime}\right]$.

13. Proposition. For any chronological set $X$, the maps $i$ and $j$ above are futurecontinuous and give an isomorphism between $\hat{X}^{p}$ and $\widehat{X^{p}}$.

Proof. We will use $\hat{\ll}$ to denote the relation in $\hat{X}$, $\hat{\ll}^{p}$ to denote the relation in $\hat{X}^{p}$ and $\widehat{\ll^{p}}$ to denote that in $\widehat{X}^{p}$.

First we will treat $i: \hat{X}^{p} \rightarrow \widehat{X^{p}}$. We have that for $x \in X\left(=X^{p}\right), i(x)=x$, and for $P \in \hat{\partial}(X), i(P)=I^{-p}[c]$ for a future chain $c$ in $X$ generating $P$; we need to see that this is well-defined. Clearly, $I^{-p}[c]$ is an IP in $X^{p}$; it is in $\hat{\partial}\left(X^{p}\right)$ because if $I^{-p}[c]=I^{-p}(x)$, then $I^{-}[c]=I^{-}(x)$, which is impossible with $P \in \hat{\partial}(X)$. We can also write it as $I^{-p}[P]$, which shows that it is independent of the chain taken as the generator for $P$.

To show $i$ is chronological, consider $x$ and $y$ in $X, P$ and $Q$ in $\hat{\partial}(X)$, and future chains $c=\left\{x_{n}\right\}$ and $d=\left\{y_{n}\right\}$ generating $P$ and $Q$ respectively:

It is largely routine to show that if $x \hat{\ll}^{p} y$, then $x \widehat{\ll^{p}} y$. Since $i(x)=x$ and $i(y)=y$, we are done.

If $x \hat{\ll}^{p} Q$, then we have $x \hat{\ll} Q$ or we have $\hat{I}^{-}(x) \neq \emptyset$ and $\hat{I}^{-}(x) \subset \hat{I}^{-}(w)$ for some $w \hat{\ll} Q$ (as above, we can take $w \in X$ ). In the first case, we have $x \in Q=I^{-}[d] \subset I^{-p}[d]$. In the second case, we have $I^{-}(x) \neq \emptyset$ and $I^{-}(x) \subset I^{-}(w)$ and $w \ll y_{n}$ for some $n$ (since $w \in Q$ ), so $x \ll^{p} y_{n}$, so again $x \in I^{-p}[d]$. In either case, this gives us $x \in i(Q)$, so $i(x) \widehat{\ll}^{p} i(Q)$, and we are done.

If $P \hat{\aleph}^{p} y$ (or $Q$ ), then we have that $P \hat{\ll} y($ or $Q)$-in which case we have $P \subset$ $I^{-}(w)$ for some $w \ll y$ (or $\left.w \in Q\right)$ - or we have that $\hat{I}^{-}(P) \neq \emptyset$ and $\hat{I}^{-}(P) \subset \hat{I}^{-}(w)$ for some $w \hat{k} y$ (or $Q$ ) -in which case we again have $P \subset I^{-}(w)$ and $w \ll y$ (or $w \in Q)$. Then in either case we have $I^{-}[c] \subset I^{-}(w)$, so $i(P)=I^{-p}[c] \subset I^{-p}(w)$, with $w \ll^{p} i(y)$ (or $\left.w \in i(Q)\right)$, so $i(P) \widehat{\ll}^{p} i(y)$ (or $i(Q)$ ), and we are done.

Now we consider $j: \widehat{X^{p}} \rightarrow \hat{X}^{p}$. For $x \in X^{p}, j(x)=x$, and for $Q \in \hat{\partial}\left(X^{p}\right)$, $j(Q)=I^{-}\left[c^{\prime}\right]$ for $c^{\prime}$ a future chain in $X$ associated to a chain $c$ in $X^{p}$ generating $Q$. Clearly $I^{-}\left[c^{\prime}\right]$ is an IP in $X$; it is in $\hat{\partial}(X)$ because if $x$ is a future limit in $X$ for $c^{\prime}$, then it is also a future limit in $X^{p}$ for $c$, which is impossible for $Q \in \hat{\partial}\left(X^{p}\right)$. We can also write it as $I^{-}[Q]$, which shows it is independent of the chain taken as the generator for $Q$ and of the chain in $X$ associated to that one. 
To show $j$ is chronological, consider $x$ and $y$ in $X, P$ and $Q$ in $\hat{\partial}\left(X^{p}\right)$, and $c=\left\{x_{n}\right\}$ and $d=\left\{y_{n}\right\}$ future chains in $X^{p}$ generating, respectively, $P$ and $Q$ :

It is routine to show that if $x \widehat{\ll^{p}} y$, then $x \hat{\kappa}^{p} y$. Thus, $j(x) \hat{\kappa}^{p} j(y)$.

If $x \widehat{\ll^{p}} Q$, then $x \in Q$. To show $j(x) \hat{\ll}^{p} j(Q)$, we must show either that $x \ll I^{-}[Q]$ or that $\hat{I}^{-}(x) \neq \emptyset$ and $\hat{I}^{-}(x) \subset \hat{I}^{-}(w)$ for some $w \ll I^{-}[Q]$, i.e., $w \in \hat{I}^{-}[Q]$. If $x \in I^{-}[Q]$, then we have $x \ll \hat{I}^{-}[Q]$. Otherwise, the only way for $x$ to be in $Q$ is for there to be some $q \in Q$ with $x \ll^{p} q$, i.e., $I^{-}(x) \neq \emptyset$ and $I^{-}(x) \subset I^{-}(w)$ for some $w \ll q$; then $\hat{I}^{-}(x) \subset \hat{I}^{-}(w)$, also. Since $w$ is in $I^{-}[Q]$, this shows $j(x) \hat{\ll}^{p} j(Q)$.

If $P \widehat{\ll^{p}} y$ (or $Q$ ), then $P \subset I^{-p}(w)$ for some $w \ll^{p} y$ (or in $Q$ ). We then have $j(P)=I^{-}[P] \subset I^{-}\left[I^{-p}(w)\right]=I^{-}(w)$. Taking the case of $y$, we need only (since $\left.I^{-}(w) \neq \emptyset\right)$ look at $I^{-}(w) \subset I^{-}(z)$ for some $z \ll y$. We have $j(P) \subset I^{-}(z)$ (and $I^{-}(j(P))=I^{-}[P]$ contains points in $P$, so is non-empty), so $j(P) \hat{\kappa}^{p} y$. For the case of $Q$, we have $w \in Q$, so for some $q \in Q, w \ll^{p} q$; again, we consider only $I^{-}(w) \subset I^{-}(z)$ for some $z \ll q$. We have $j(P) \subset I^{-}(z)$ with $z \in I^{-}[Q]$, i.e., $z \hat{\ll} j(Q)$; thus, $j(P) \hat{\aleph}^{p} j(Q)$.

Now we consider the compositions: For $P \in \hat{\partial}(X), j(i(P))=I^{-}\left[I^{-p}[P]\right]=$ $I^{-}[P]=P$. Thus, we have $j \circ i=1_{\hat{X}^{p}}$. For $Q \in \hat{\partial}\left(X^{p}\right), i(j(Q))=I^{-p}\left[I^{-}[Q]\right]$. This is clearly contained in $Q$; we must show it the same as $Q$. For $q \in Q$, we know there is some $p \in Q$ with $q \ll^{p} p$, i.e., either $q \ll p$ (in which case we immediately have $\left.q \in I^{-}\left[I^{-}[Q]\right] \subset I^{-p}\left[I^{-}[Q]\right]\right)$ or $I^{-}(q) \neq \emptyset$ and $I^{-}(q) \subset I^{-}(w)$ for some $w \ll p$. In the latter case, we can find $z$ with $w \ll z \ll p$, so that we have $q \ll^{p} z \ll p$, yielding $q \in I^{-p}\left[I^{-}[Q]\right]$. This gives us $i \circ j=1_{\widehat{X^{p}}}$.

Now we know that $i$ and $j$ provide an isomorphism of sets-with-a-relation between $\hat{X}^{p}$ and $\widehat{X^{p}}$. Since future limits are defined purely in terms of the relations, we automatically get that both maps are future-continuous.

It is tempting to think of the maps $i_{X}$ and $j_{X}$, as collections for all $X$ in PconPdisChron, as forming a pair of natural transformations $\mathbf{i}: \mathbf{p} \circ{ }^{\widehat{\lambda}} \rightarrow \widehat{ } \widehat{\wedge} \mathbf{p}$ and $\mathbf{j}: \widehat{\wedge} \mathbf{p} \rightarrow \mathbf{p} \circ{ }^{\wedge}$; since they are inverses of one another, we would then have a natural isomorphism between the two constructions. This would then establish that all that was done with the construction of $\widehat{X^{p}}$ can also be done with the construction of $\hat{X}^{p}$. Since this latter is the construction that precisely reproduces the GKP future causal boundary, this is a desirable outcome. However, there is a problem: One cannot compose the functors in the order $\mathbf{p} \circ^{-}{ }^{-}$, since Proposition 6 allows us to conclude that $\hat{f}: \hat{X} \rightarrow \hat{Y}$ is future-continuous (starting with $f: X \rightarrow Y$ future-continuous) only if $Y$ is already past-determined. But there is a way around this difficulty, thereby establishing the categorical nature of the GKP construction:

We will use $X^{+}$to denote $\hat{X}^{p}$, for $X$ any chronological set. For a futurecontinuous function $f: X \rightarrow Y$ between chronological sets, we cannot in general consider $\hat{f}^{p}$, since $\hat{f}$ is not in general future-continuous unless $Y$ is pastdetermined. But so long as $Y$ is past-distinguishing and $X$ is past-connected, we can use $\widehat{f^{p}}: \widehat{X^{p}} \rightarrow \widehat{Y^{p}}$, which is future-continuous; then we can define $f^{+}=$ $j_{Y} \circ \widehat{f^{p}} \circ i_{X}: X^{+} \rightarrow Y^{+}$. Finally, we define $\iota_{X}^{+}=j_{X} \circ \iota_{X}^{\uparrow}: X \rightarrow X^{+}$.

14. Theorem. For any chronological set $X, X^{+}$is past-determined and futurecomplete, and $\iota_{X}^{+}: X \rightarrow X^{+}$is future-continuous; if $X$ is past-connected (or, respectively past-distinguishing), then so is $X^{+}$. If $X$ is past-connected and $Y$ is a past-distinguishing chronological set, then for any future-continuous map $f: X \rightarrow$ 
$Y, f^{+}: X^{+} \rightarrow Y^{+}$is the unique future-continuous map such that $f^{+} \circ \iota_{X}^{+}=\iota_{Y}^{+} \circ f$. If $Y$ is also past-determined and future-complete, than $f^{+}: X^{+} \rightarrow Y$ is the unique future-continuous map such that $f^{+} \circ \iota_{X}^{+}=f$.

Proof. We need to establish that several diagrams involving the injections $\iota$ commute. Some of these follow from categorical principles when in the category PconChron, but others involve the particularities of these maps (especially when pastconnectedness is not assumed).

One noncategorical result is the application of future-completion using the full strength of Proposition 6: The purely categorical use of future completion applies only to the category of past-determined, past-distinguishing chronological sets (as per summary statement (3)), but we need to apply it to the map $\iota_{X}^{p}: X \rightarrow X^{p}$, where it would be pointless to assume $X$ past-determined. But Proposition 6 gives us the result anyway, as displayed in Diagram 1:

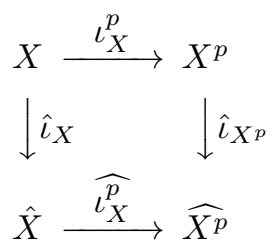

\section{Diagram 1. composition is $\iota_{X}^{\uparrow}$}

The map $\iota_{X}^{p}$ is always future-continuous, as is the standard future injection $\hat{\iota}$, whether for $X$ or for $X^{p}$. By Proposition 6, we can define future completion of $\iota_{X}^{p}$, and have it be future-continuous, even without $X^{p}$ being past-distinguishing, so long as for any future chain $c$ in $X$ generating an element of $\hat{\partial}(X), \iota_{X}^{p}[c]$ has no more than one future-limit in $\widehat{X^{p}}$; but this is manifestly the case, since $\hat{\partial}\left(X^{p}\right)$ (or $\hat{\partial}$ in general) is defined in such a way that no two elements can have identical pasts. Proposition 6 also yields that Diagram 1 commutes, and, as indicated, the composition is $\iota_{X}^{\uparrow}$.

For $X$ past-connected, an entirely categorical result comes from starting with $\hat{\iota}_{X}: X \rightarrow \hat{X}$ and applying the past-determination functor and the natural transformation $\iota^{\mathbf{p}}$, yielding the commutative diagram of future-continuous maps shown in Diagram 2.

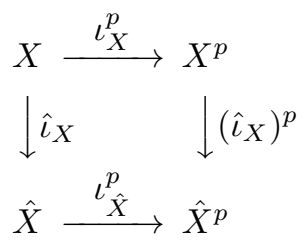

Diagram 2. composition is $\iota_{X}^{+}$(see Diagram 4$)$

Even if $X$ is not past-connected, Diagram 2 still commutes (since each composition is just inclusion), and $\left(\hat{\iota}_{X}\right)^{p}$ is still future-continuous: For chronologicality, 
we need to show that if $x \ll^{p} y$ (in $X$ ), then $x \hat{\aleph}^{p} y$ (in $\hat{X}$ ): If $I^{-}(x) \subset I^{-}(w)$ for $w \ll y$, then $\hat{I}^{-}(x) \subset \hat{I}^{-}(w)$ and $w \hat{\ll} y$. For future-continuity, we need to show that if $x$ is a future limit of a future chain $c$ in $X^{p}$, then $x$ is also a future limit of $c$ in $\hat{X}^{p}$ : If $x$ is a future limit of $c$ in $X^{p}$, then by Lemma $9 x$ is a future limit of an associated future chain $c^{\prime}$ in $X$; application of the future-continuous $\hat{\iota}_{X}$ shows that $x$ is a future limit of $c^{\prime}$ also in $\hat{X}$; then, applying Lemma 9 once more (with $c^{\prime}$ being associated to $c$ in $\hat{X}$ as well as in $X$ ), we have $x$ a future limit of $c$ in $\hat{X}^{p}$.

The identification of the composition in Diagram 2 as $\iota_{X}^{+}$will follow from Diagram 4. But before that we must consider Diagram 3: This commutes because both $i_{X} \circ\left(\hat{\iota}_{X}\right)^{p}$ and $\hat{\iota}_{X^{p}}$ are just inclusion on the set-level. Commuting in the other direction, with $j_{X}$, then follows automatically.

$$
\begin{aligned}
& X^{p}=X^{p} \\
& \left.\downarrow \hat{\iota}_{X}\right)^{p} \quad \downarrow_{\hat{\iota}_{X}} \\
& \hat{X}^{p} \underset{i_{X}}{\stackrel{i_{X}}{\longrightarrow}} \widehat{X^{p}}
\end{aligned}
$$

\section{DiAGRAM 3}

The final result involving commuting injections is shown in Diagram 4. To establish the commutativity, we first combine Diagrams 2 and 3 into Diagram 5 and compare with Diagram 1; we use the uniqueness property of future completion from Proposition 6 (summary statement (1)) to see that that the bottom lines of Diagrams 1 and 5 must be the same, and that is the content of Diagram 4 . (As before, establishing the result with $i_{X}$ automatically yields the result for $j_{X}$ ). Combining this with Diagram 1 and the definition of $\iota_{X}^{+}$yields the identification alluded to in Diagram 2.

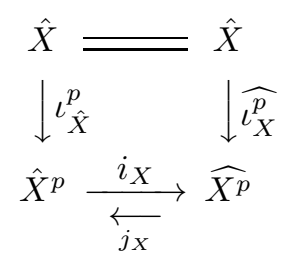

\section{Diagram 4}

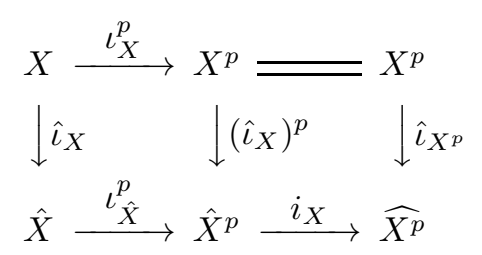

Diagram 5. Diagrams 2 and 3 combined 
Finally, in Diagram 6 we consider the diagram derived from future-continuous $f: X \rightarrow Y$ for $X$ past-connected and $Y$ past-distinguishing. The top two portions of Diagram 6 commute due to previously established results (summary statements (1) and (4)). The bottom portion commutes, as that is the definition of $f^{+}$. The overall structure of Diagram 6 can be expressed as Diagram 7 .

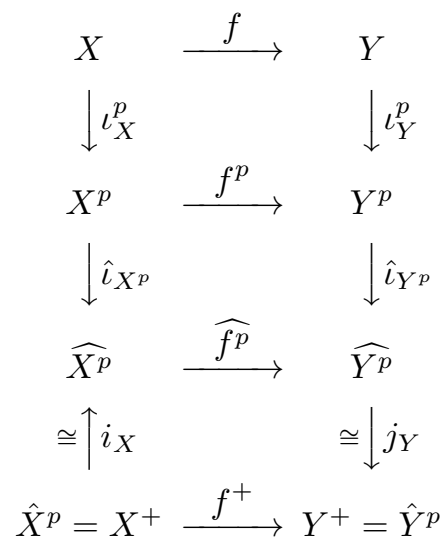

Diagram 6

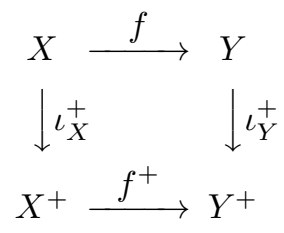

Diagram 7. overall structure of Diagram 6

The map $f^{+}$is unique for fulfilling its role in Diagram 7 , since for any other future-continuous map $g: X^{+} \rightarrow Y^{+}$making Diagram 7 commute, $i_{Y} \circ g \circ j_{X}$ would fill the role of $\widehat{f^{p}}$ in Diagram 6 , and the uniqueness portion of summary statement (4) would show $i_{Y} \circ g \circ j_{X}=\widehat{f^{p}}$, so $g=f^{+}$.

If $Y$ is also past-determined and future-complete, then $Y^{+}=Y$ and $\iota_{Y}^{+}=1_{Y}$, yielding the last statement in the theorem.

Theorem 14 enables us to define the functor + : PconPdisChron $\rightarrow$ FcplPdetPconPdisChron, with $X^{+}=\hat{X}^{p}$ and $f^{+}: X^{+} \rightarrow Y^{+}$, even though we cannot define $f^{+}$as $\hat{f}^{p}$. We also have the natural transformation $\boldsymbol{\iota}^{+}: \mathbf{I} \rightarrow \mathbf{U}^{+} \circ+$ (where I denotes the identity on PconPdisChron) yielding + as left adjoint to $\mathbf{U}^{+}$. Since a functor can have only one left adjoint, up to natural isomorphism, there must be a natural isomorphism, then, between + and ${ }^{-} \circ \mathbf{p}$. This is provided,

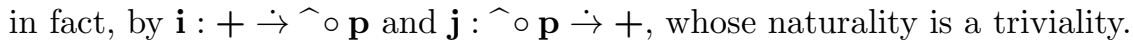

Expressing this in summary form as before, we have

(10) For $X$ in PconChron, $Y$ in PdisChron, and $f: X \rightarrow Y$ future-continuous, $f^{+}: X^{+} \rightarrow Y^{+}$is the unique future-continuous map with $f^{+} \circ \iota_{X}^{+}=\iota_{Y}^{+} \circ f$. 
(11) For $X$ in PconChron, $Y$ in FcplPdetPdisChron, and $f: X \rightarrow Y$ future-continuous, $f^{+}: X^{+} \rightarrow Y$ is the unique future-continuous map with $f^{+} \circ \iota_{X}^{+}=f$.

(12) Restricted to PconPdisChron, the construction of $X^{+}, f^{+}$, and $\iota_{X}^{+}$is functorial, natural, and universal, hence, the unique way to fulfill (10) and (11).

For a spacetime $M, \hat{M}^{p}$ is precisely the addition to $M$ of the GKP future causal boundary: $\hat{M}$ is identified with the IPs of $M$ (the TIPs - IPs not of the form $I^{-}(x)$ for any point $x$-being $\hat{\partial}(M)$ ), and the $\ll^{p}$ relation is exactly that as specified by GKP. Since any strongly causal spacetime $M$ is in PconPdisChron, any "reasonable" way of future-completing $M$-an embedding of $M$ into a past-determined, pastdistinguishing, future-complete object-must contain the GKP future boundary in the sense of Theorem 14: there is a unique future-continuous extension of the embedding to the GKP future completion of $M$.

Typically, one applies these ideas by starting with a strongly causal spacetime $M$ and a purported future completion of it, such as a topological embedding of $M$ into another spacetime $N, j: M \rightarrow N$, which preserves the chronological relations of $M$ and which includes future endpoints for all the endless timelike curves in $M$. The actual object which is the purported future completion of $M$ is the closure of $j(M)$ in $N$; call this object $Y$, with a chronology relation inherited from $N$. Then, since $M$ is past-connected and $Y$ (typically) is past-distinguishing, we have, from Theorem 14, $j^{+}: M^{+} \rightarrow Y^{+}$; assuming $Y$ is itself future-complete, we have $Y^{+}=Y^{p}$. Then, in particular, $j^{+}$maps $\hat{\partial}(M)$ to the boundary of $j[M]$ in $N$. If we had the good sense to choose the enveloping spacetime $N$ in such a way that every point in the closure of $j[M]$ was actually a future endpoint of a timelike curve in $M$ (mediated by $j$ ) - or if we restrict $Y$ to be just such points - then $Y$ is mapped onto by $j^{+}$, with the boundary-points of $Y$ being covered by $j^{+}[\hat{\partial}(M)]$. This places strong restrictions on just what $Y$ can be.

As an example, consider a spacetime $M$ which is conformal to $K \times \mathbb{L}^{1}$, where $K$ is any compact Riemannian manifold (recall $\mathbb{L}^{n}$ denotes Minkowski $n$-space). $M$ is globally hyperbolic (any $K \times\{t\}$ is a Cauchy surface), hence, past-determined. The future chronological boundary of $M$ consists of a single point $i^{+}$which is the future limit of every endless timelike curve. (This can be seen by observing that for any point $p \in M$ and any future-endless timelike curve $c, c$ eventually enters the future of $p$; this is best noted by looking at projections into the factors $K$ and $\mathbb{L}^{1}$.) Thus, for any past-distinguishing, past-determined, future-complete chronological set $Y$ and any future-continuous map $f: M \rightarrow Y, f$ has a unique future-continuous extension to $M \cup\left\{i^{+}\right\}$; this means precisely that there is some point $y^{+} \in Y$ such that for every endless timelike curve $c$ in $M, y^{+}$is the future limit of $f \circ c$. In other words, any "reasonable" way of putting a future boundary on $M$ essentially replicates the GKP procedure, producing $i^{+}$and nothing more (any other points in $Y$ which are neither in $f[M]$ nor $y^{+}$are not the future limit of anything in $f[M]$ and are in that sense disconnected from $M$ ).

More generally, for $M$ any strongly causal spacetime, let $f: M \rightarrow Y$ be any future-continuous map into a past-distinguishing, future-complete, chronological set. Applying the past-determination functor we have $f^{p}: M^{p} \rightarrow Y^{p}$, a future continuous map into a past-determined, past-distinguishing, future-complete chronological set. Thus, we have the future extension of $f^{p}$ to $f^{+}: M^{+} \rightarrow Y^{p}$. Since 
$\hat{\partial}(M)$ (the GKP future causal boundary of $M$ ) contains the future limit of every future-endless timelike curve in $M$, the same is true of its image in $Y^{p}$ under $f^{+}$; assuming that $f$ is an embedding of $M$ into $Y$, this means that the portion of $Y$ which is directly connected to $f[M]$-i.e., the points of $Y$ which are future limits of chains coming from $M$-consists, aside from $f[M]$ itself, of a set future-continuously mapped onto by the GKP future causal boundary of $M$.

\section{Section IV: Generalized Conditions and Results}

In a spacetime, for every point $x, I^{-}(x)$ is an IP; this is not necessarily so in a chronological set $X$. A future chain $c$ may not have a future limit because the point $x$ which one might nominate for the future limit of $c$, has a decomposable past. However, this can still play the role of a future limit in a more generalized sense. Let us order by inclusion the IPs contained within $I^{-}(x)$ (for any $x \in X$ ); if $P$ is maximal in this ordering, call it a past component of $x$ (so if $I^{-}(x)$ is an IP, there is only that one past component). Let us call $x$ a generalized future limit of a future chain $c$ if $I^{-}[c]$ is a past component of $x$. As before, if $x$ is a generalized future limit of $c$, then it's a generalized future limit of any sub-chain of $c$; conversely, if it's a generalized future limit of some sub-chain of $c$, then it's a generalized future limit of $c$. Call $X$ generalized past-distinguishing if whenever $x$ and $y$ in $X$ share a past component, $x=y$ (this is a stronger condition than being past-distinguishing). If $X$ is generalized past-distinguishing, a future chain can have no more than one generalized future limit. Call $X$ generalized future-complete if every future chain has a generalized future limit (a weaker condition than being future-complete). Call a chronological function $f: X \rightarrow Y$ generalized future-continuous if for every generalized future limit $x$ of a future chain $c$ in $X, f(x)$ is a generalized future limit of $f[c]$ (this is neither a stronger nor a weaker property than being futurecontinuous); a composition of generalized future-continuous functions is generalized future-continuous. Finally, call a point $x \in X$ generalized past-determined if $I^{-}(x)$ is non-empty and for any $w$ with $I^{-}(w)$ containing some past component of $x$, $x \ll y$ for all $y \gg w$ (a stronger property than being past-determined); and $X$ is generalized past-determined if all of its points are.

These generalized notions are what are needed to provide the mappings into chronological sets with decomposable pasts for some of the points; a typical example would be starting with a spacetime and embedding it into another manifold to create a boundary for the spacetime, with some of the boundary points possibly having decomposable pasts. We still get useful information, such as analogues of Proposition 7, Corollary 12, and Theorem 14-allowing a comparison with the GKP future causal boundary - so long as the envelopment obeys these generalized notions:

15. Theorem. For any generalized future-continuous map $f: X \rightarrow Y$ with $X$ a past-connected chronological set and $Y$ a generalized past-determined, generalized past-distinguishing, generalized future-complete chronological set, there are unique generalized future-continuous maps $\hat{f}: \hat{X} \rightarrow Y, f^{p}: X^{p} \rightarrow Y$, and $f^{+}: X^{+} \rightarrow Y$ such that $\hat{f} \circ \hat{\iota}_{X}=f^{p} \circ \iota_{X}^{p}=f^{+} \circ \iota_{X}^{+}=f$.

Proof. First consider $\hat{f}$ : For any $P \in \hat{\partial}(X)$, with $c$ a future chain generating $P, \hat{f}[c]=f[c]$ must have a unique generalized future limit $y \in Y$, so $\hat{f}(P)=$ $y$ is forced. For $x \ll P$, with $P$ generated by $c$, we have $x \in P$, so $\hat{f}(x)=$ 
$f(x) \in f[P]=f\left[I^{-}[c]\right] \subset I^{-}[f[c]]$, which is a past component of $\hat{f}(P)$; that implies $I^{-}[f[c]] \subset I^{-}(\hat{f}(P))$, so $\hat{f}(x) \ll \hat{f}(P)$. For $P \ll x$, with $P$ generated by $c$, we have $I^{-}[c] \subset I^{-}(w)$ for some $w \ll x$, which implies $c \subset I^{-}(w)$ (as in the proof of Proposition 6); therefore, $I^{-}[f[c]] \subset I^{-}(f(w))$ and $f(w) \ll f(x)$. With $\hat{f}(P)$ the generalized future limit of $f[c]$, we know that $I^{-}[f[c]]$ is a past component of $\hat{f}(P)$; thus, since $\hat{f}(P)$ is generalized past-determined, we have $\hat{f}(P) \ll f(x)=\hat{f}(x)$. For $P \ll Q$, we interpolate $P \ll x \ll Q$. Thus, $\hat{f}$ is chronological. Generalized future-continuity follows from that of $f$, since the only new future limits are those in $\hat{\partial}(X)$.

Next consider $f^{p}$ : For $x \ll^{p} y$ in $X$, we have $I^{-}(x) \subset I^{-}(w)$ for some $w \ll y$. We also have, since $X$ is past-connected, that $x$ is a future limit of some future chain $c$, so $I^{-}[c] \subset I^{-}(w)$. Thus, $I^{-}[f[c]] \subset I^{-}(f(w))$ and $f(w) \ll f(y)$. Since $f$ is generalized future-continuous, $f(x)$ is the generalized future limit of $f[c]$, i.e., $I^{-}[f[c]]$ is a past component of $f(x)$. Since $f(x)$ is generalized past-determined, this gives us $f(x) \ll f(y)$. Therefore, $f^{p}$ is chronological. For generalized futurecontinuity, the proof in Proposition 11 suffices, once we obtain an extension of Lemma 9 , that $x$ is a generalized future limit of a future chain $c$ in $X^{p}$ if and only if $x$ is a generalized future limit of any associated chain $c^{\prime}$ in $X$ :

With $I^{-}\left[c^{\prime}\right]$ a past component of $x$ in $X$, we need to have $I^{-p}[c]$ a past component of $x$ in $X^{p}$ (the other way being easy). The proof of Lemma 9 shows that $I^{-p}[c] \subset$ $I^{-p}(x)$, we just need to show it a maximal IP with that property. Suppose $d$ is a chain in $X^{p}$ with $I^{-p}[c] \subset I^{-p}[d] \subset I^{-p}(x)$; then $I^{-}\left[c^{\prime}\right] \subset I^{-}\left[d^{\prime}\right] \subset I^{-}(x)$, where $d^{\prime}$ is a chain in $X$ associated to $d$. Since $I^{-}\left[c^{\prime}\right]$ is maximal with the property of being an IP contained in the past of $x$, we have $I^{-}\left[c^{\prime}\right]=I^{-}\left[d^{\prime}\right]$, and it follows that $I^{-p}[c]=I^{-p}[d]$.

Finally, $f^{+}$comes from the previous two constructions, just as in Theorem 14.

As an example, let us consider the spacetime $M$ formed from $\mathbb{L}^{2}$ by deleting two timelike half-lines: $L_{0}=\{(0, t) \mid t \leq 0\}$ and $L_{2}=\{(2, t) \mid t \geq 2\}$. Then $\hat{\partial}(M)$ consists of these IPs (illustrated in figure 1): for each $t \leq 0, P_{t}^{L}$, bounded by $L_{0}$ and the null line from $(0, t)$ going to the left in the past, and $P_{t}^{R}$, bounded by $L_{0}$ and the null line from $(0, t)$ going to the right in the past; for each $t>2, Q_{t}^{L}$, bounded by $L_{2}$, the null line from $(2, t)$ going to the left in the past, and the null line from $(2,2)$ going to the right in the past, and $Q_{t}^{R}$, bounded by $L_{2}$, the null line from $(2, t)$ going to the right in the past, the null line going from $(2,2)$ to $(0,0)$, and $L_{0}$; $Q_{2}$, bounded by the null line from $(2,2)$ going to the right in the past, the null line going from $(2,2)$ to $(0,0)$, and $L_{0}$; and the usual future causal boundary for $\mathbb{L}^{2}$, consisting of the pasts of null lines going out to future infinity, as well as the entire spacetime. In $\hat{M}$, we have $P_{0}^{R} \ll(3,4)$ (because $\left.\hat{I}^{-}\left(P_{0}^{R}\right) \subset I^{-}(3,3)\right)$, but there is no relation between $P_{0}^{L}$ and $(3,4)$. Also, since there is no relation between $(1,1)$ and $(3,4)$, the space is not past-determined (same for $M)$. The past-determination of $\hat{M}$ adds some relations: $(s, s) \ll^{p}(3,4)$ for $0<s<2$; however, even in $\hat{M}^{p}$, there is no relation between $P_{0}^{L}$ (or $(x, t)$ for $x<0$ and $t<x$ ) and $(3,4)$.

A typical embedding of $M$ to create a boundary for it would be to use $\hat{M}$, except to coalesce $P_{0}^{R}$ and $P_{0}^{L}$ into a point $P_{0}$, with $P_{0}$ not related to $(3,4)$; call this $\bar{M}$. This is not past-determined, since $(1,1)$ is not related to $(3,4)$. We can add relations to $\bar{M}$ by saying that two points are related if there is a curve between them, causal in $\mathbb{L}^{2}$, somewhere timelike, allowed to include "one side" of 
$L_{0}$ or $L_{2}$ but not to cross either of them (the points $P$ and $Q$ being identified with one side or the other of those two lines). This space, $\overline{\bar{M}}$, is past-determined, pastdistinguishing, and generalized future-complete (since $P_{0}$ has two past components, it is not future-complete); hence we get a generalized future-continuous map from $M^{+}$to $\overline{\bar{M}}$; this is just the map coalescing $P_{0}^{R}$ and $P_{0}^{L}$ into $P_{0}$. Similarly, any means of adding complete boundaries to the slits in $M$ will end up being a quotient of the boundaries from $\hat{M}$.

\section{Appendix: An Example with the Full Causal Boundary}

Why has the foregoing structure been involved solely with the future chronological boundary and not with some combination of future and past, such as the complete causal boundary of the GKP construction? In a nutshell, the reason is that the combined causal boundary, future with past, is, in general, neither futurenor past-complete: It's as if one were to attempt a completion of a Riemannian manifold by adding endpoints to all finite-length endless curves, and then one found, for instance, that the added endpoints themselves constituted a finite-length endless curve. Here is an example of how that can happen with the GKP causal boundary and a rather ordinary spacetime (illustrated in figure 2):

Our spacetime $M$ will be a subset of Minkowski 2-space, $\mathbb{L}^{2}$, in which we will cut an infinite number of slits and poke an infinite collection of holes. First we select three parallel null lines as reference objects, say $L^{+}=\{t=x+1\}, L^{0}=\{t=x\}$, and $L^{-}=\{t=x-1\}$; we also select a future-timelike curve $c$ asymptotic (in the future) to the middle null line, say $c(s)=(\sinh s, \cosh s)$. For each positive integer $k$, consider a short null segment centered at $c(2 k)$, parallel to the reference lines, say from $(\sinh (2 k)-.5, \cosh (2 k)-.5)$ to $(\sinh (2 k)+.5, \cosh (2 k)+.5)$; denote the past and future endpoints of these null segments by, respectively, $p_{k}$ and $q_{k}$. To form $M$, delete from $\mathbb{L}^{2}$ each vertical segment from $p_{k}$ down to $L^{-}$, each vertical segment from $q_{k}$ up to $L^{+}$, and each point $c(n)$, all $k$ and all $n$.

The future causal boundary for $M$ includes an IP $P_{n}$ corresponding to each of the deleted points $c(n)$, while the past causal boundary includes an IF $F_{n}$ for the same. (The future causal boundary also has, for each of the slits, IPs for each point of the left and right sides of the slit, except a single IP for the bottom point; an IP for future timelike infinity, $i^{+}$; and IPs for future null infinity, $\Im^{+}$- but with points corresponding to the null lines between $L^{-}$and $L^{+}$missing from the usual $\Im^{+}$for $\mathbb{L}^{2}$, due to the slits. The past causal boundary also has, for each slit, IFs for each point of the left and right sides, except a single IF for the top; and past timelike and null infinity, $i^{-}$and $\Im^{-}$. However, we shall be largely unconcerned with these other points in the causal boundary.) We have each $P_{2 k} \subset P_{2 k+1}$, so that within $\hat{M}, P_{2 k} \ll P_{2 k+1}$, but no other relations obtain among these boundary points, due to the slits; and the future chronological completion of $M$ is precisely the same as adding the GKP future causal boundary to $M$. Similarly, within $\check{M}$, the past chronological completion of $M$ (the same as adding the GKP past causal boundary), we have $F_{2 k-1} \ll F_{2 k}$ (since $F_{2 k-1} \supset F_{2 k}$ ) but no other relations among those particular boundary points.

There is more than one scheme for combining future and past causal boundaries into a single causal boundary (see, for instance, [S1] and [S2] for a good alternative to the GKP prescription), but they all agree in a spacetime as simple as this one: Each $P_{n}$ is identified with $F_{n}$, which we'll call $z_{n}$ (and the two copies of the left side 
of each slit - the one from the future causal boundary and the one from the past causal boundary - are identified, and the same with the right sides, with a single boundary point for the top and one for the bottom of each slit); this is called $M^{*}$ in [HE]. Thus, in any choice of chronology relation on $M^{*}$ which makes the obvious mappings from $\hat{M}$ and $\check{M}$ to $M^{*}$ chronological, we must have $z_{2 k-1} \ll z_{2 k}$ (from $\check{M})$ and $z_{2 k} \ll z_{2 k+1}($ from $\hat{M})$, so that $\left\{z_{n}\right\}$ is a future chain in $M^{*}$. However, this chain has no future limit in $M^{*}$-for instance, the past of $L^{0}$ is not an IP, since the slits decompose that past into an infinite number of past sets: In essence, the chain $\left\{z_{n}\right\}$ "escapes" our intended completion of $M$ by aiming for the "hole" in $\Im^{+}$left by the missing points corresponding to the null lines between $L^{-}$and $L^{+}$.

Szabados, in section 6 of [S1], proposes a chronology relation on $M^{*}$ that, in this case, would leave no relations at all among the $\left\{z_{n}\right\}$; this construction has the advantage of not adding any additional relations among the points of $M$-unlike the GKP construction in the future or past causal boundaries, or the future and past completions defined here- but the disadvantage of not preserving relations already defined in $\hat{M}$ or $\check{M}$.

\section{REFERENCES}

[GKP] R. P. Geroch, E. H. Kronheimer, and R. Penrose, Ideal points in space-time, Proc. Roy. Soc. Lond. A 327 (1972), 545-67.

[HE] S. W. Hawking and G. F. R. Ellis, The Large Scale Structure of Space-Time, Cambridge University, Cambridge, 1973.

[KP] E. H. Kronheimer and R. Penrose, On the structure of causal spaces, Proc. Camb. Phi. Soc. 63 (1967), 481-501.

[M] S. Mac Lane, Categories for the Working Mathematician, Springer-Verlag, New York, 1971.

[S1] L. B. Szabados, Causal boundary for strongly causal spacetimes, Class Quantum Grav. 5 (1988), 121-134.

[S2] L. B. Szabados, Causal boundary for strongly causal spacetimes: II, Class Quantum Grav. 6 (1989), 77-91.

Department of Mathematics, Saint Louis University, St. Louis, MO 63103, USA

E-mail address: harrissg@slu.edu 


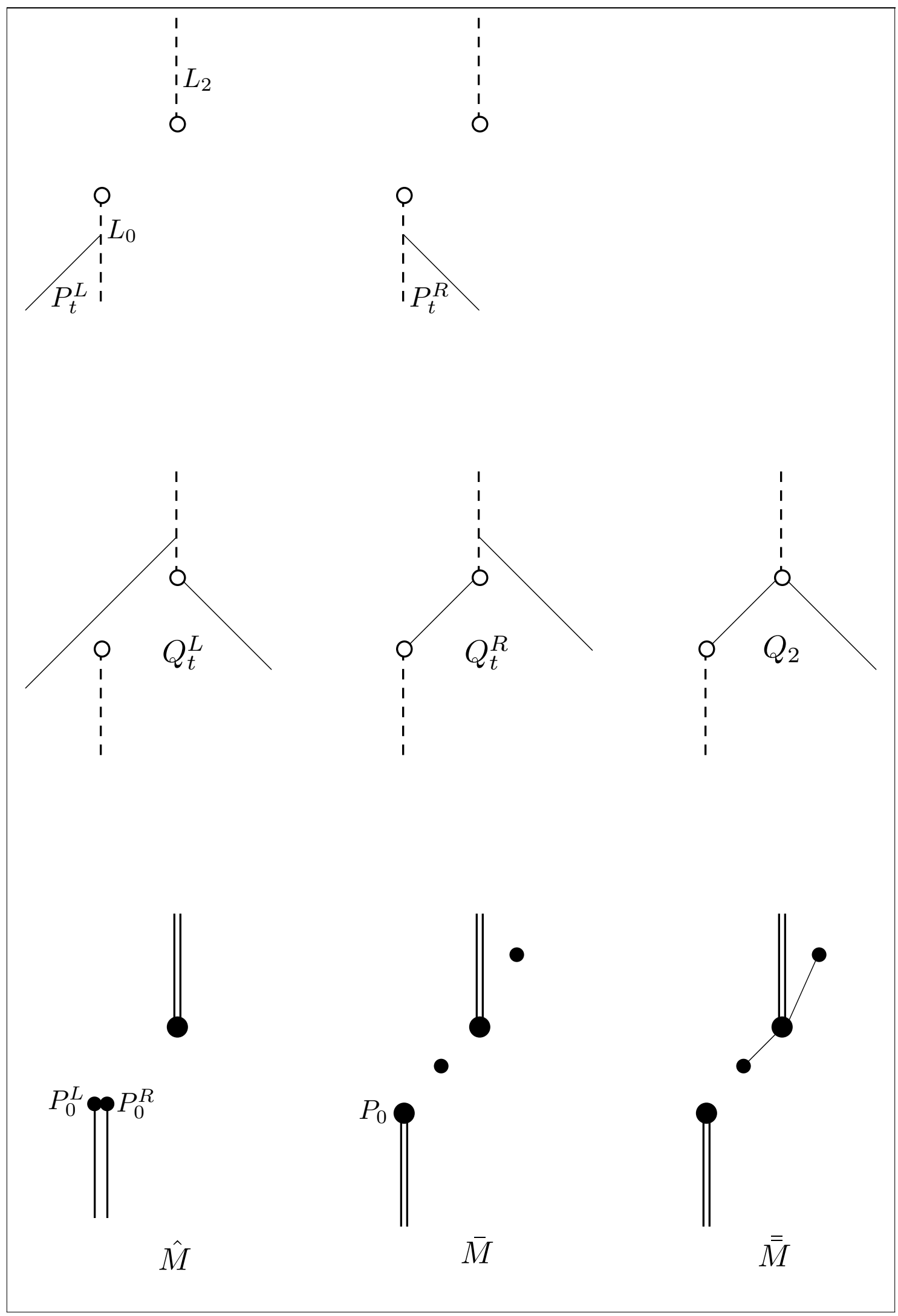




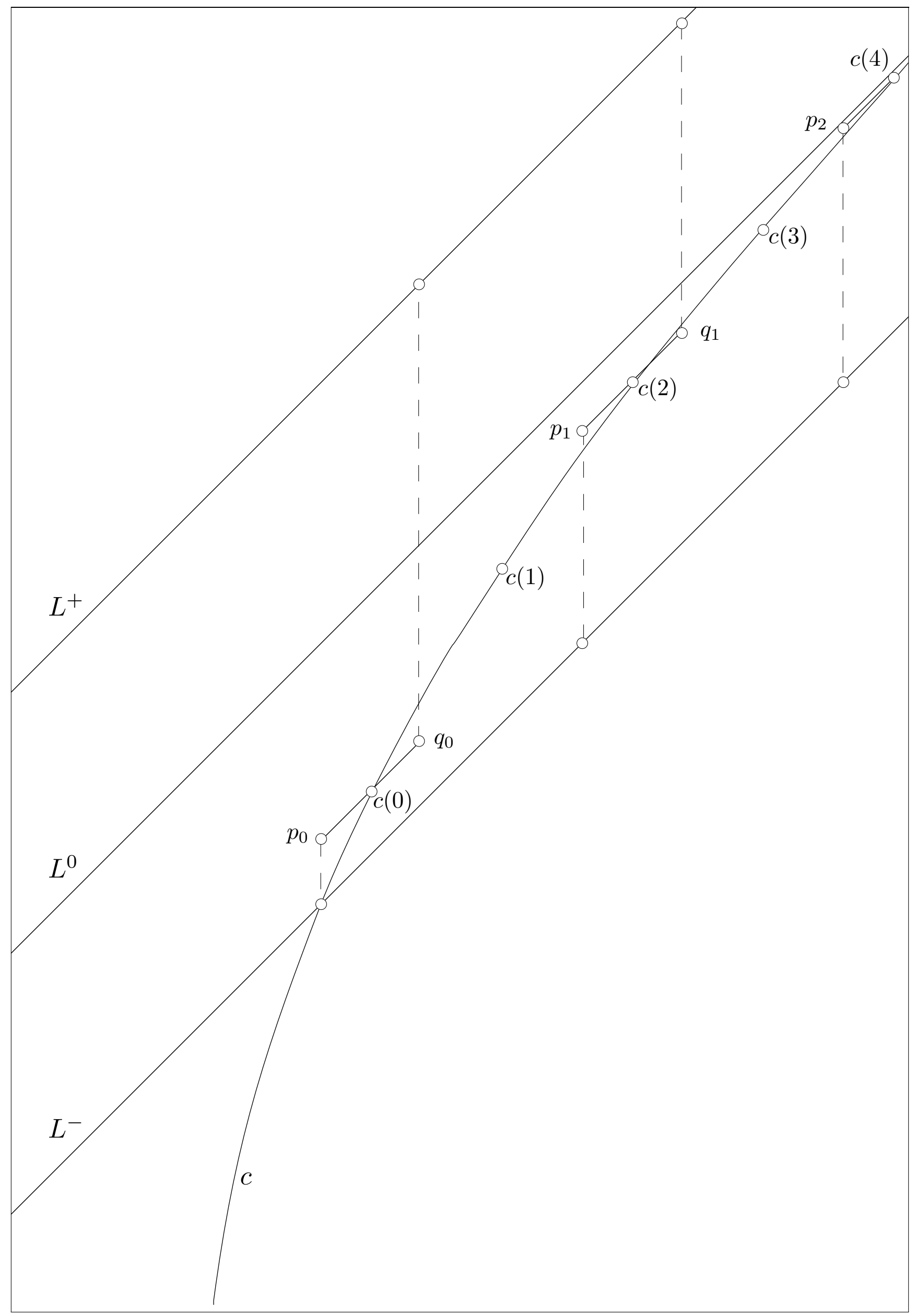

